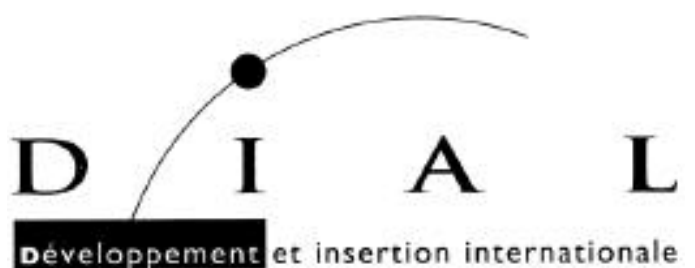

Développement et insertion internationale

DOCUMENT DE TRAVAIL

DT/2000/11

\title{
Taux de rendement de l'éducation sur le marché du travail d'un pays en développement
}

Florence A restoff 


\title{
Taux de rendement de l'éducation sur le marché du travail d'un pays en développement
}

\author{
Un réexamen du modèle de gains de Mincer
}

Florence Arestoff* *

\section{Résumé :}

Cet article propose une analyse des rendements éducatifs issue du modèle de Mincer (1974). Dans le cas de Madagascar où plus de 50\% des élèves étaient scolarisés dans une école privée en 1997, le type d'éducation reçue (publique ou privée) est pris en compte, tandis que les biais potentiels de sélection sur le marché du travail et d'endogénéité du niveau d'études sont corrigés.

Sous l'hypothèse d'un marché du travail concurrentiel, l'éducation ne semble exercer aucun impact direct sur les revenus. Elle ne servirait donc que d'indicateur de la motivation et des facilités de chacun à travailler. Ce résultat est confirmé dans le secteur formel, lorsque l'on suppose que le marché du travail de la capitale malgache est segmenté. En revanche, dans le secteur informel, le taux de rendement d'une année d'éducation supplémentaire atteint $7 \%$ et l'enseignement public engendre un revenu du travail significativement plus élevé que l'enseignement privé.

\section{Abstract :}

This article analyses the returns of education as defined by Mincer's model (1974), in the malagasy case. Selection bias in the labour market and endogeneity bias of education are corrected for. Moreover, the type of education is taken into account. Indeed, this variable is suspected to play a key role as more than $50 \%$ of the pupils were enrolled in private schools in 1997.

Under a competitive labour market hypothesis, education doesn't seem to exert any direct impact over revenues. So, it would only be an indicator of motivation and abilities of each to work. This result is confirmed in the formal sector, when we suppose a segmented labour market in Antananarivo. On the contrary, in the informal sector, the rate of return of one more year of education reaches $7 \%$ and public education allows work revenues significatively higher than private education does.

- DIAL, Université Paris IX-Dauphine

- Adresse postale : 2, Avenue de Versailles, 75016 Paris E-mail : florence_arestoff@hotmail.com 


\section{Table des matières}

1. REVENUS, NIVEAU ET TYPE D'ÉDUCATION : ANALYSES DESCRIPTIVES ................................6

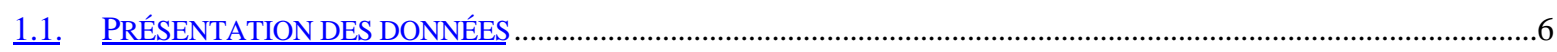

1.2. REVENUS HORAIRES ET NIVEAU D'ÉDUCATION ………………………………….....................................

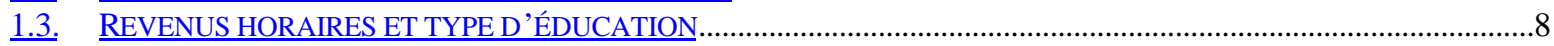

2. MODELISATION DES RENDEMENTS ET DE L'EDUCATION ….............................................................9

2.1. L'ÉQUATION DE MINCER (1974) ET SES LIMITES …………………………………...................................10

2.2. AMÉLIORATION DU MODĖLE DE GAINS DE MINCER : LA DISTINCTION ENTRE ÉDUCATION PUBLIQUE ET

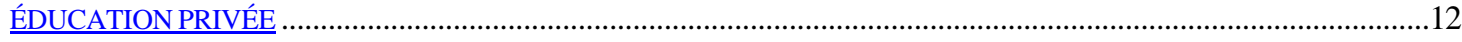

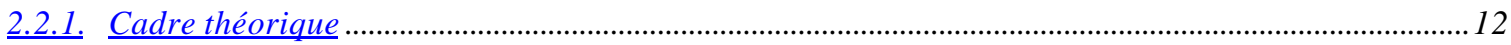

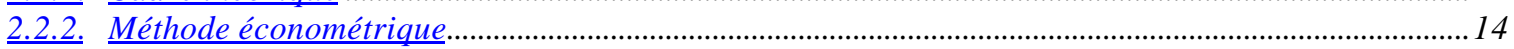

3. TAUX DE RENDEMENT DE L'ÉDUCATION, SUR UN ECHANTILLON D'HOMMES...................15

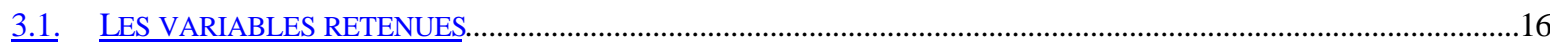

3.2. ESTIMATION DE L'ÉQUATION DE MINCER ET DE SES PREMIÈRES EXTENSIONS ................................................19

3.3. INSTRUMENTATION DU NIVEAU D'ÉTUDES ET INTÉGRATION DU TYPE D’ÉDUCATION DANS L'ÉOUATION DE

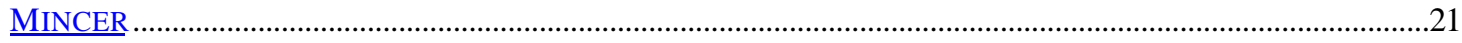

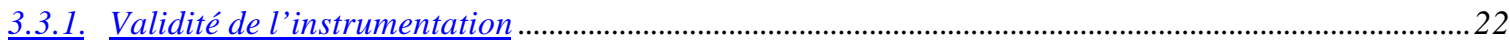

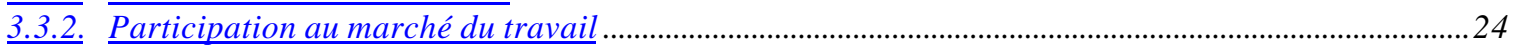

3.3.3. Revenus et taux de rendement de l'éducation .......................................................................................2

4. TAUX DE RENDEMENT DE L'ÉDUCATION SOUS L'HYPOTHÈSE D'UNE SEGMENTATION

DU MARCHÉ DU TRAVAIL .............................................................................................................................................................26

4.1. THÉORIE DE LA SEGMENTATION DU MARCHÉ DU TRAVAIL : UNE BRÈVE PRÉSENTATION ...............................26

4.2. REVENUS DU TRAVAIL DANS LES SECTEURS FORMEL ET INFORMEL : ANALYSE DESCRIPTIVE …………….....27

4.2.1. Revenus horaires et niveau d'éducation, dans les secteurs formel et informel .....................................2 28

4.2.2. Revenus horaires et type d'éducation, dans les secteurs formel et informel ..........................................28

4.3. TAUX DE RENDEMENT DE L'ÉDUCATION DANS LES SECTEURS FORMEL ET INFORMEL ....................................29

4.3.1. Affectation dans le secteur formel ……………………………………………………………….... 30

4.3.2. Taux de rendement de l'éducation ............................................................................................................. 30

4.3.3. Effet du type d'éducation et des autres variables................................................................................... 32

4.3.4. Pertinence de l'hypothèse d'une segmentation du marché du travail : test de Chow et analyse de la

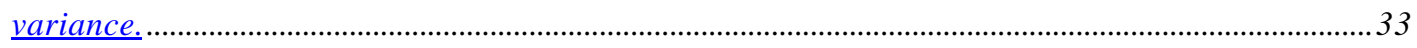

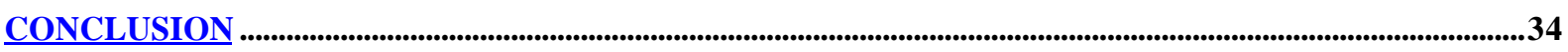

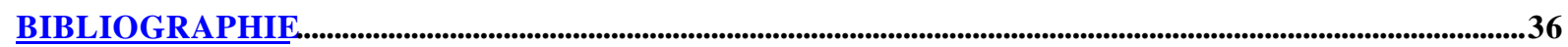

\section{Table des illustrations}

TABLEAU 1: REVENU HORAIRE MOYEN ET NIVEAU D'ÉTUDES, EN 1997 ……………………………………….....

TABLEAU 2: REVENU HORAIRE MOYEN ET TYPE D'ÉDUCATION, PAR NIVEAU D'ÉTUDES, EN 1997.............................9

TABLEAU 3： EQUATIONS DE REVENUS DES HOMMES, EN 1997 …………………………………………………....20

TABLEAU 4: MODĖLE DE GAINS CORRIGÉ DU BIAIS DE SÉLECTIVITÉ, AVEC NIVEAU D'ÉTUDES INSTRUMENTÉS, POUR LES HOMMES, EN 1997 ……………………………………………………………………...2

TABLEAU 5: REVENU HORAIRE MOYEN ET NIVEAU D'ÉTUDES, EN 1997, DANS LES SECTEURS FORMEL ET

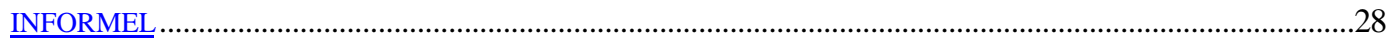

TABLEAU 6: REVENU HORAIRE MOYEN ET TYPE D'ÉDUCATION, PAR NIVEAU D'ÉTUDES, EN 1997, DANS LES SECTEURS FORMEL ET INFORMEL ………………………………………………………………...2

TABLEAU 7: ESTIMATION DES REVENUS DANS LES SECTEURS FORMEL ET INFORMEL, EN 1997 ………………………...31 


\section{Table des graphiques}

GRAPHIQUE 1: SYNTHÈSE DE LA PROCÉDURE D'ESTIMATION DES REVENUS ............................................................22 GRAPHIQUE 2: EFFET TOTAL DU TYPE D'ÉDUCATION SUR LA PARTICIPATION AU MARCHÉ DU TRAVAIL DES

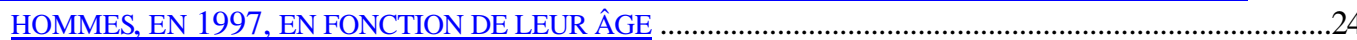




\title{
Taux de rendement de l'éducation sur le marché du travail d'un pays en développement
}

\author{
Un réexamen du modèle de gains de Mincer
}

Florence Arestoff

Les investissements éducatifs, comme tous les investissements, peuvent être évalués en termes de taux de rendement. La théorie du capital humain [Mincer (1974) et Becker (1975)] fournit une méthodologie qui permet d'estimer l'accroissement des revenus individuels résultant d'une année d'études supplémentaire. Or, on peut se demander si une année d'éducation publique génère les mêmes bénéfices sur le marché du travail qu'une année d'éducation privée, toutes choses égales par ailleurs ? Il s'agit là d'une question à laquelle la littérature empirique a peu répondu directement, et souvent dans un contexte de pays développés.

En effet, si les études s'attachant aux conséquences de la qualité de l'éducation sur les revenus se sont fortement développées depuis une vingtaine d'années, elles portent essentiellement sur les Etats-Unis. La qualité scolaire est le plus souvent représentée par des indicateurs d'offre tels que le ratio élèves par maître, la formation des enseignants ou leur niveau de rémunération. Néanmoins, Betts (1995), Grogger (1996) et Bedi and Garg (2000) ont montré que l'établissement fréquenté constituait aussi une variable de première importance dans la détermination des revenus et ce, indépendamment des indicateurs de qualité scolaire habituellement retenus. Un tel résultat nous conduit donc à nous interroger sur la possibilité que le taux de rendement de l'éducation soit surestimé lorsque le type d'éducation n'est pas retenu parmi les variables explicatives des revenus.

Face à ce problème, Madagascar constitue une étude de cas intéressante. Alors qu'à la fin des années 70, le pays jouissait encore d'un avantage comparatif en matière d'éducation par rapport aux autres pays africains, depuis le début des années 80 , il ne peut plus assurer ni le développement ni le maintien d'un système éducatif public de qualité. Avec la récession économique, les dépenses publiques d'éducation ont considérablement chuté, provoquant une notable désaffection de l'éducation publique au profit de l'éducation privée. 
Dans une première section de ce chapitre, nous présentons les données et quelques statistiques descriptives afin d'établir une première approche de la relation entre le niveau d'éducation, le type d'éducation (public ou privé), et les revenus. Dans une seconde section, nous proposons une modélisation des rendements de l'éducation visant à enrichir le modèle de Mincer (1974) selon lequel les revenus ne sont fonction que du niveau d'éducation et de l'expérience professionnelle. Dans la troisième section, diverses analyses économétriques portant sur les revenus du travail sont menées sur un échantillon d'hommes. Comme Glewwe (1996) et Jarousse et Mingat (1986) ${ }^{1}$, nous procédons par étapes dans l'amélioration de l'estimation du taux de rendement de l'éducation. Enfin, dans une dernière section, ces mêmes analyses sont réalisées sur deux sous-populations dissociées de travailleurs des secteurs formel et informel car les caractéristiques de ces deux secteurs conduisent à penser qu'il existe une segmentation du marché du travail à Antananarivo.

\section{REVENUS, NIVEAU ET TYPE D'ÉDUCATION : ANALYSES DESCRIPTIVES}

Le modèle de base sous-jacent à la présente analyse est celui du capital humain. Le gain d'un actif occupé sera donc expliqué avant tout par ses caractéristiques propres, et notamment son niveau d'éducation, son expérience professionnelle, et dans une large mesure par son capital social, héritage du savoir familial. Par conséquent, l'estimation du taux de rendement de l'éducation implique de disposer d'une base de données relativement riche. Celle que nous utilisons est issue de deux enquêtes auprès des ménages de l'agglomération d'Antanarivo, réalisées par le Projet MADIO² entre Avril et Juin 1997.

\subsection{Présentation des données}

D'une manière générale mais peut-être plus encore dans le cadre d'un pays en développement tel que Madagascar, l'analyse des revenus tirés de l'activité est un aspect du marché du travail qui est complexe à étudier pour plusieurs raisons. D'abord parce que, traditionnellement, la question des revenus constitue un thème sensible pour la population qui se montre souvent réticente à répondre sincèrement sur sa situation financière. Ensuite, parce qu'il est difficile d'obtenir des informations fiables dans ce domaine. Ces dernières nous sont fournies par la phase 1 (Emploi) de «l'enquête 1-2-3»³ qui porte sur l'emploi de 3000 ménages à

auxquels nous empruntons d'ailleurs le sous-titre « Un réexamen du modèle de Mincer » DIAL-INSTAT-ORSTOM

Elaborée pour traiter de la mesure économique du secteur informel, l'enquête 1-2-3 a été mise en œuvre au Cameroun en 1993 et 1995 puis à Madagascar. Il s'agit d'une enquête auprès des ménages urbains qui se décompose en trois 
Antananarivo. L'enquête Emploi permet de recueillir des informations sur l'activité, le chômage et les revenus. Elle s'adresse aux individus âgés de dix ans et plus qui appartiennent à un échantillon de ménages représentatifs de la population tananarivienne. Cette enquête prend en compte non pas les salaires des individus mais le montant mensuel de leur rémunération. Il s'agit d'une distinction importante quand on sait que le pourcentage de salariés est de 52,6\% à Antanarivo ${ }^{4}$ et que très peu d'actifs possèdent une comptabilité écrite, notamment dans la petite agriculture familiale et dans le secteur informel urbain. De plus, les individus qui ne veulent pas ou ne souhaitent pas donner d'informations sur leur revenu mensuel sont incités à le faire dans une question plus indirecte où on leur demande de se situer dans des tranches de revenus. Cette information est alors utilisée pour imputer un revenu mensuel à tous les actifs occupés 5 .

Parallèlement, nous utilisons l'enquête SET97 ${ }^{6}$. Réalisée en Mai et Juin 1997, elle porte sur un échantillon de 1022 ménages (soient 4522 individus), tirés de façon aléatoire parmi les 3000 ménages interrogés un mois plus tôt sur l'emploi. Le laps de temps séparant les deux enquêtes est suffisamment court pour que la confrontation des deux bases de données soit possible. Outre un grand nombre de caractéristiques socio-économiques, l'enquête SET97 fournit une information détaillée sur l'historique de la scolarisation de chaque individu. Ainsi, tous les établissements fréquentés en primaire et en secondaire sont recensés pour chaque membre du ménage âgé d'au moins cinq ans, ce qui permet notamment de rapporter chacune des années d'études à un type d'établissement particulier, public ou privé.

\subsection{Revenus horaires et niveau d'éducation}

Selon Projet MADIO (1997), à Antananarivo, plus de la moitié des actifs perçoivent moins de 123000 Fmg par mois en 1997, montant correspondant au salaire minimum des catégories les moins qualifiées, en vigueur au premier trimestre 1997. Mais le revenu moyen cache une très forte disparité de situation notamment chez les non-salariés. Ainsi, les aides familiaux ne perçoivent, en général, aucune rémunération. De même, alors que dans le secteur informel, le revenu mensuel moyen est de 127000 Fmg en 1997, il atteint 233000 Fmg dans le secteur formel.

phases. La première phase est une enquête sur l'emploi, adaptée au contexte des pays en développement. Elle permet de caractériser le marché du travail mais également de repérer les unités de production informelles qui seront enquêtées dans la seconde phase. La consommation des ménages, considérée notamment sous l'angle de la demande exprimée auprès du secteur informel, fait l'objet d'une troisième phase. Seule la première phase sera donc utilisée ici. Sur l'ensemble du pays, ce taux ne dépasse pas $15 \%$.

Nous utilisons le revenu mensuel calculé par le Projet MADIO.

SET $=$ Sante-Education-Transferts . 
Afin de déterminer s'il existe une relation, au moins apparente, entre l'éducation et les revenus, nous limitons notre échantillon aux individus âgés d'au moins 18 ans qui, pour la plupart, ont terminé leurs études. Nous excluons aussi les migrants ${ }^{7}$ dont le comportement est supposé trop différent de celui des non-migrants sur le marché du travail.

Par simplification, les revenus que nous analysons ne concernent que l'activité principale. Nous ne tenons pas compte des revenus perçus dans l'activité secondaire, bien que le taux de pluri-activité atteigne 34,2\% à Madagascar.

Sachant que dans le secteur informel, où sont employés 59,5\% des actifs occupés en 1997, les heures de travail ne sont, par définition, pas réglementées, nous choisissons d'analyser les revenus horaires des travailleurs ${ }^{8}$. Le revenu horaire est préféré aux revenus mensuel et annuel car ceux-ci ne parviennent pas à isoler le coût de transaction du travail, les questions d'arbitrage travail-loisir et des effets plus transitoires du chômage. De plus, lors de l'analyse économétrique du revenu, nous serons ainsi dispensés d'instrumenter l'offre de travail individuelle, c'est-à-dire le nombre d'heures travaillées.

Tableau 1 : Revenu horaire moyen et niveau d'études, en 1997

\begin{tabular}{ccccc}
\hline \hline en Fmg & $\begin{array}{c}\text { Sans éducation ou } \\
\text { Primaire }\end{array}$ & Secondaire 1 ${ }^{\text {er }}$ cycle & Secondaire 2 ${ }^{\text {nd }}$ cycle & Supérieur \\
\hline Echantillon total & 675 & 806 & 1173 & 2295 \\
Hommes & 878 & 928 & 1232 & 2547 \\
Femmes & 434 & 685 & 1087 & 1889 \\
\hline \hline
\end{tabular}

Source : Enquête SET97, enquête 1-2-3 phase1 (1997), nos propres calculs.

Le tableau 1 indique que le revenu horaire moyen croît avec le niveau d'études mais aussi que ses rendements sont croissants. Ainsi, alors que passer du primaire au $1^{\text {er }}$ cycle du secondaire génère une croissance du revenu de $19 \%$, cette croissance atteint $96 \%$ lorsque l'on passe du $2^{\text {nd }}$ cycle du secondaire au cycle supérieur.

Quel que soit le niveau d'études atteint, le revenu moyen des femmes demeure très inférieur à celui des hommes et ce, plus particulièrement pour des individus de niveau primaire. Les hommes gagnent alors deux fois plus que les femmes en moyenne, par heure travaillée.

\subsection{Revenus horaires et type d'éducation}

Sur ce même échantillon, Arestoff (2000) montre qu'avec la crise économique, la rémunération du premier emploi est devenue significativement différente selon le type d'éducation reçue. On pourrait alors penser que cette différence se vérifie aussi en 1997, pour

\footnotetext{
7 Sont considérés comme migrants les individus nés hors de l'agglomération d'Antananarivo.
} 
l'ensemble des travailleurs, quelle que soit l'avancement de leur carrière professionnelle. Or, il n'en est apparemment rien.

Tableau 2 : Revenu horaire moyen et type d'éducation, par niveau d'études, en 1997

\begin{tabular}{|c|c|c|c|c|c|c|}
\hline \multirow[b]{2}{*}{ en Fmg } & \multicolumn{2}{|c|}{ Primaire } & \multicolumn{2}{|c|}{ Secondaire $1^{\mathrm{er}}$ cycle } & \multicolumn{2}{|c|}{ Secondaire $2^{\text {nd }}$ cycle ou Supérieur } \\
\hline & Public & Privé & Public & Privé & Public & Privé \\
\hline Echantillon total & 685 & 681 & 832 & 825 & 1523 & 1758 \\
\hline Hommes & 902 & 867 & 1037 & 912 & 1449 & 2108 \\
\hline Femmes & 405 & 515 & 612 & 754 & 1627 & 1147 \\
\hline
\end{tabular}

Source : Enquête SET97, enquête 1-2-3 phase1 (1997), nos propres calculs.

Note : Pour la population ayant suivi des études supérieures, le type d'éducation reçu ne prend pas en considération les établissements supérieurs fréquentés.

Sur l'échantillon total, le tableau 2 montre peu de différences entre les revenus horaires perçus par les anciens élèves du public et ceux du privé.

En revanche, lorsque l'on analyse distinctement les deux sexes, les différences de revenus par type d'éducation se creusent. Pour les hommes les plus éduqués (études de $2^{\text {nd }}$ cycle du secondaires ou études supérieures), l'éducation privée génère un revenu moyen qui est supérieur à celui de l'éducation publique alors que pour de plus faibles niveaux d'études, l'éducation publique apparaît relativement plus profitable. Le phénomène strictement inverse se vérifie chez les femmes.

Ces résultats nous conduisent donc à supposer que le comportement sur le marché du travail des hommes diffère suffisamment de celui des femmes pour que le taux de rendement de l'éducation des uns soit estimé indépendamment de celui des autres. Notre problème consistera alors à savoir si ce taux de rendement par sexe varie significativement lorsque l'on suppose que les revenus ne sont pas seulement fonction du niveau mais aussi du type d'éducation.

\section{MODELISATION DES RENDEMENTS ET DE L'EDUCATION}

Le modèle standard de capital humain [Mincer (1974) et Becker (1975)] suppose que les salariés sont rémunérés à leur productivité marginale et que celle-ci augmente avec le capital humain accumulé. Le bénéfice net d'une année de scolarisation supplémentaire peut alors être estimé à partir de données portant sur les revenus d'individus dont le niveau d'éducation diffère. Nous envisageons ci-après un certain nombre d'enrichissements de la procédure standard afin de réduire les biais potentiels d'estimation des rendements de l'éducation.

8 Comme l'enquête renseigne sur le nombre d'heures travaillées par jour au cours de la semaine précédente, nous pouvons aisément en déduire un revenu horaire, en supposant que ce nombre d'heures vaille pour les 4,3 semaines qui composent un mois. 


\subsection{L'équation de Mincer (1974) et ses limites}

Pour déterminer le taux de rendement de S années d'éducation, Mincer (1974) estime une équation de la forme :

$\ln Y_{S}=c+r S+a E+b E^{2}+u$

avec $\mathrm{Y}_{\mathrm{S}}$, le revenu individuel,

$\mathrm{S}$, le nombre d'années d'études,

E, l'expérience professionnelle. Elle prend une forme quadratique afin de représenter la concavité du profil de gains due à l'investissement postscolaire en capital humain, c'est-à-dire les rendements décroissants de l'expérience,

c, la constante, que les théoriciens du capital humain interprètent comme le salaire de base sans capital humain,

et $\mathrm{u}$, un terme stochastique, représentant les facteurs non observés qui affectent le revenu. Il s'agit de facteurs de moyenne nulle, que l'individu ne connaît pas forcément.

Dans cette équation de gains semi-logarithmique, si u est distribué selon les propriétés standard, alors l'estimation de r par la méthode des MCO correspond au taux de rendement privé (par opposition au taux de rendement social) de l'éducation. Il mesure l'augmentation du revenu $\left(\mathrm{Y}_{\mathrm{S}}-\mathrm{Y}_{\mathrm{S}-1}\right)$ résultant d'une année d'éducation supplémentaire rapportée au coût annuel de cet investissement scolaire ${ }^{9}$, soit :

$\frac{Y_{S}-Y_{S-1}}{Y_{S-1}}=\frac{e^{c+r S+a E+b E^{2}+u}}{e^{c+r(S-1)+a E+b E^{2}+u}}-1=e^{r}-1 \cong r$

Bien que Mincer (1993) considère que la scolarisation et l'expérience expliquent à elles seules un tiers de la variance des taux de salaire dans les pays occidentaux, il existe un certain nombre de limites du modèle de Mincer (1974).

D'une part, il est naturel de supposer que l'environnement parental influe sur la valeur économique du temps passé à l'école. Selon Bowles (1972), en raison de l'influence de l'environnement familial sur le niveau d'études atteint, omettre le niveau d'éducation des parents ou leur activité professionnelle provoquerait la surestimation du rendement de l'éducation.

La littérature tant économique que sociologique offre de nombreuses démonstrations de l'impact positif de l'éducation et du revenu des parents sur le développement cognitif de

9 Le modèle de Mincer (1974) suppose que les coûts d'une année supplémentaire d'éducation sont exclusivement composés de coûts d'opportunité, c'est-à-dire de gains auxquels l'individu renonce en allant à l'école. L'estimation du taux de rendement de l'éducation fait donc abstraction des coûts directs à la charge de l'individu (frais d'inscription, d'uniformes, de transport scolaire, etc.). 
l'enfant, sur la qualité de l'éducation qu'il reçoit et sur sa réussite éducative. Or, le cadre d'apprentissage que des parents éduqués peuvent offrir à leur enfant lui permettra aussi d'accroître sa productivité pour un nombre d'années d'études donné (Murnane et al., 1981). Parallèlement, le niveau d'éducation des parents matérialise le capital social de l'individu. Grâce à un père éduqué, l'individu pourra profiter de réseaux de connaissance qui lui permettront peut-être d'occuper des postes plus valorisants en termes de position, de rémunération, etc. Le népotisme et la stratification sociale remettent alors en question les mécanismes du marché. Selon Bowles (1972), une classe sociale et un niveau d'éducation ne déterminent pas un revenu mais plutôt une opportunité. Celle-ci correspond au fait de choisir parmi des emplois différents, chaque emploi se caractérisant par divers aspects monétaires et non monétaires. Le revenu reçu par un individu est alors le résultat d'un choix, contraint par l'ensemble des opportunités d'emplois offertes.

L'ensemble de ces facteurs nous conduit donc à supposer que l'environnement parental exerce à la fois un effet direct sur les revenus de l'individu et un effet indirect transitant par son niveau d'éducation.

D'autre part, supposer que la relation entre les revenus et l'éducation est linéaire revient à supposer que les chocs d'offre et de demande de travail ont les mêmes effets sur le taux de rendement de l'éducation à tous les niveaux d'études. Heckman et al. (1996) rejettent cette hypothèse, arguant que la durée de la scolarité (S) n'est pas homogène, toutes les années n'ayant pas forcément la même efficacité. Heckman and Polachek (1974) ont ainsi mis en évidence l'existence d'un effet-diplôme selon lequel les années de scolarité ne débouchant pas sur l'obtention d'un diplôme généreraient un rendement plus faible que les autres.

Afin d'autoriser le rendement de l'éducation à varier avec le volume de l'investissement éducatif, il est donc préférable d'intégrer le niveau d'études sous une forme quadratique comme le font Angrist and Lavy (1997).

Ces phénomènes pris en compte, le modèle de Mincer «étendu » s'écrit alors :

$\ln Y_{S}=c+r_{1} S+r_{2} S^{2}+a E+b E^{2}+d X+u(\mathbf{3})$

avec $X$, un vecteur de caractéristiques individuelles et parentales.

Alors $\frac{\partial \ln Y_{S}}{\partial S}=r_{1}+2 r_{2} S$ correspond au taux de rendement marginal de l'éducation dans lequel on anticipe que $r_{2}$ est négatif, matérialisant ainsi des rendements marginaux décroissants. 


\subsection{Amélioration du modèle de gains de Mincer : la distinction entre éducation publique et éducation privée}

Si le type d'éducation, public ou privé, agit sur les revenus à la fois indirectement par la réussite scolaire [comme le montrent Arestoff et Bommier (2000)] mais aussi directement, alors le modèle de Mincer «étendu » [équation (3)] tend encore à surestimer le taux de rendement de l'éducation. Pour y remédier, nous proposons de poursuivre notre adaptation du modèle de Mincer.

\subsubsection{Cadre théorique}

Supposons un individu i. En intégrant le fait d'avoir reçu une éducation publique ou privée $\left(\mathrm{T}_{\mathrm{i}}\right)$ parmi les variables explicatives de ses revenus, l'équation (3) peut être réécrite comme suit :

$\ln Y_{i}=\alpha+r_{1} S_{i}+r_{2} S_{i}^{2}+\beta_{1} T_{i}+\beta_{2} E_{i}+\beta_{3} E_{i}^{2}+\beta_{4} X_{i}+\varepsilon_{1 i}$

avec $\alpha$, la constante et $\varepsilon_{1 \mathrm{i}}$, le résidu.

L'estimation de cette équation par des MCO n'est possible que si les variables explicatives ne sont pas corrélées avec les facteurs non observés. Or, les rendements de l'éducation peuvent varier entre les individus, en fonction de paramètres propres à chacun d'eux qui sont à même d'influer sur les revenus (et l'éducation) mais sont omis de l'équation : facilités intrinsèques, motivation à étudier, préférence pour le présent, accès aux sources de financement, etc.

Comme l'ont notamment montré Ichino et Winter-Ebmer (1999), le taux de rendement de l'éducation sera surestimé si les individus qui disposent de davantage de facilités intrinsèques sont enclins à suivre de plus longues études mais sont aussi enclins à recevoir de plus hauts revenus. Leur supplément de revenu serait alors attribué à tort à leur supplément d'éducation. Dans ce cas, on parle de biais d'endogénéité ${ }^{10}$.

Inversement, les possibles erreurs de mesure non aléatoires sur les variables explicatives ou sur la variable dépendante peuvent générer un biais à la baisse dans l'estimation du taux de rendement de l'éducation. Dans l'enquête que nous utilisons, les revenus sont appréhendés par la question suivante :

10 Griliches (1977) a montré que ce biais d'endogénéité ne pouvait être positif que si l'hypothèse de neutralité posée par Ben-Porath (1967) était remise en cause. En effet, selon cette hypothèse, le capital humain initial est également productif sur l'acquisition de connaissances et sur le marché du travail. Cette hypothèse impliquerait donc un biais de facilité négatif car l'accumulation de capital humain étant coûteuse, les individus dotés de plus grandes facilités initiales devraient investir relativement moins dans l'éducation. En relâchant l'hypothèse de neutralité, on suppose donc que les facilités des individus sont plus productives si elles sont investies dans la formation plutôt que directement utilisées sur le marché du travail. 
« Dans votre emploi principal, combien avez-vous gagné le mois dernier (ou à combien estimez-vous vos revenus pour ce travail?»

Les enquêteurs ne vérifiant pas les feuilles de paye des salariés et les travailleurs informels estimant eux-mêmes leurs gains, les revenus sont probablement évalués avec une certaine marge d'erreur. D'après Brownstone and Valletta (1996), des événements tels que le chômage ou la formation continue, qui affectent le nombre d'heures travaillées, sont aussi à même de générer des réponses biaisées aux questions d'enquêtes sur les revenus.

De même, lorsque le véritable niveau d'éducation $\mathrm{S}$ est mesuré avec une erreur aléatoire $\varepsilon$ telle que $S^{\prime}=S+\varepsilon$, tous les paramètres estimés de l'équation de gains sont biaisés. Ils le seront d'autant plus que de multiples variables sont introduites dans l'équation afin de contrôler les autres sources possibles de biais [Ashenfelter and Krueger (1994) et Griliches (1997)]. Dans l'échantillon de Behrman et al. (1996), le pourcentage d'erreurs dans le report des années d'éducation atteint 9,3\%, par exemple ${ }^{11}$.

Pour éliminer ces biais, la solution la plus fréquemment retenue consiste à instrumenter correctement la variable $\mathrm{S}_{\mathrm{i}}$ et son carré, c'est-à-dire à caractériser un déterminant (ou instrument) approprié de l'éducation qui ne soit pas corrélé avec le résidu des revenus. Le processus d'instrumentation comprend deux étapes. D'abord, on estime l'effet des variables instrumentales sur les variables endogènes ; puis on estime l'effet des variables instrumentales sur les revenus. Puisque, par hypothèse, les instruments ne sont corrélés avec les revenus que parce qu'ils influent sur l'éducation, alors le ratio de l'effet de l'instrument sur les revenus par rapport à son effet sur l'éducation fournit une estimation de l'effet causal de l'éducation sur les revenus.

Le modèle peut donc s'écrire :

$\ln Y_{i}=\alpha+r_{1} S_{i}+r_{2} S_{i}^{2}+\beta_{1} T_{i}+\beta_{2} E_{i}+\beta_{3} E_{i}^{2}+\beta_{4} X_{i}+\varepsilon_{1 i}$

$S_{i}=\gamma Z_{1 i}+\varepsilon_{2 i}$

$S_{i}^{2}=\delta Z_{2 i}+\varepsilon_{3 i}$

où $Z_{i}$ désigne l'ensemble des variables explicatives de $S_{i}$ et de $S_{i}{ }^{2}$.

11 Sous l'hypothèse d'une terme résiduel normalement distribué, les problèmes d'estimation causés par les erreurs de mesure dans un modèle de régression linéaire sont limités : si l'erreur porte sur une variable indépendante, elle biaisera tous les coefficients estimés vers zéro ; si l'erreur porte sur la variable dépendante, elle augmentera l'écarttype des résidus mais ne biaisera pas les paramètres estimés. 
Les termes d'erreurs sont tels que :

$$
\left(\begin{array}{l}
\boldsymbol{\varepsilon}_{1 i} \\
\boldsymbol{\varepsilon}_{2 i} \\
\boldsymbol{\varepsilon}_{3 i}
\end{array}\right) \approx N\left(\left[\begin{array}{l}
0 \\
0 \\
0
\end{array}\right],\left[\begin{array}{lll}
\boldsymbol{\sigma}_{11} & \boldsymbol{\sigma}_{12} & \boldsymbol{\sigma}_{13} \\
\boldsymbol{\sigma}_{21} & \boldsymbol{\sigma}_{22} & \boldsymbol{\sigma}_{23} \\
\boldsymbol{\sigma}_{31} & \boldsymbol{\sigma}_{32} & \boldsymbol{\sigma}_{33}
\end{array}\right]\right)
$$

Après insertion de (5) et (6) dans (4), on obtient la forme réduite du modèle :

$$
\begin{aligned}
& \ln Y_{i}=\alpha+r_{1} \gamma Z_{1 i}+r_{2} \delta Z_{2 i}+\beta_{1} T_{i}+\beta_{2} E_{i}+\beta_{3} E_{i}^{2}+\beta_{4} X_{i}+r_{1} \varepsilon_{2 i}+r_{2} \varepsilon_{3 i}+\varepsilon_{1 i} \text { (7) } \\
& S_{i}=\gamma Z_{1 i}+\varepsilon_{2 i} \\
& S_{i}^{2}=\delta Z_{2 i}+\varepsilon_{3 i}
\end{aligned}
$$

Nous considérons que l'instrumentation du niveau d'études corrige aussi le biais de sélection du type d'éducation reçue. Cela revient donc à supposer qu'au moment d'opter pour tel ou tel établissement, l'individu lui-même ou ses parents ne prennent pas en considération l'impact possible de cet établissement au delà de la scolarité.

\subsubsection{Méthode économétrique}

L'équation initiale que nous cherchons à estimer est l'équation (4).

Nous ne connaissons le revenu de l'individu que si le salaire offert est supérieur à son salaire de réservation. Or, rien ne laisse supposer que les travailleurs rémunérés constituent un échantillon aléatoire de la population active dans son ensemble. Au contraire, on peut penser que les individus qui ne travaillent pas (et notamment les femmes) font état de caractéristiques particulières : nombreux enfants en bas âge, milieu social favorisé ou au contraire défavorisé, etc. Le taux de rendement de l'éducation pouvant s'en trouver affecté, les estimations de $\ln Y_{i}$ qui utilisent les revenus observés doivent alors prendre en compte la censure à gauche de l'échantillon, comme démontré par Heckman (1974).

Afin de corriger le biais de participation ou non au marché du travail, nous estimons un modèle TOBIT en suivant la procédure en deux étapes d'Heckman (1979). Ce faisant, l'objectif est d'obtenir une évaluation des gains potentiels ou plus précisément de la productivité non observée de chaque individu, y compris de ceux qui ne perçoivent pas de revenus.

Parallèlement, dans l'estimation de $\ln Y_{i}$, nous instrumentons le niveau d'éducation et son carré. La procédure d'instrumentation permet de résoudre les problèmes de convergence des MCO dans la situation générique où les variables explicatives sont corrélées avec les résidus. 
La procédure est théoriquement assez simple. Il suffit, dans une première étape, d'estimer $\mathrm{S}_{\mathrm{i}}$ et $S_{i}^{2}$ en régressant $S_{i}$ sur $Z_{1 i}$ et $S_{i}^{2}$ sur $Z_{2 i}, Z_{1 i}$ et $Z_{2 i}$ figurant dans les équations (5) et (6). Dans une seconde étape, les valeurs estimées de $S_{\mathrm{i}}$ et de $\mathrm{S}_{\mathrm{i}}{ }^{2}$ sont utilisées pour expliquer la variable dépendante $\left(\ln \mathrm{Y}_{\mathrm{i}}\right)$. Comme le niveau d'éducation et le revenu sont estimés par des $\mathrm{MCO}$, on parle de Double Moindres Carrés (DMC) ${ }^{12}$.

Le modèle prend alors la forme d'un système de quatre équations :

$\left\{\begin{array}{l}P_{i} A R T_{i}=\psi Z_{3 i}+v_{i} \\ S_{i}=\gamma Z_{1 i}+\varepsilon_{2 i} \\ S_{i}^{2}=\delta Z_{2 i}+\varepsilon_{3 i}\end{array}\right.$

$\ln Y_{i}=\alpha+r_{1} \hat{S}_{i}+r_{2} \hat{S}_{i}^{2}+\beta_{1} T_{i}+\beta_{2} E_{i}+\beta_{3} E_{i}^{2}+\beta_{4} X_{i}+\beta_{5} \lambda_{i}+\varepsilon_{1 i}$

avec $\mathrm{PART}_{\mathrm{i}}$, la probabilité de participer au marché du travail pour l'individu $\mathrm{i}$,

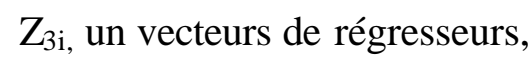

et $\lambda_{\mathrm{i}}$, l'inverse du ratio de Mills calculé à partir de l'équation Probit (8). La théorie du capital humain permet d'interpréter $\lambda_{\mathrm{i}}$ comme le capital humain «non observé » ayant permis à l'individu i de surmonter les aléas et les exigences du marché du travail.

\section{TAUX DE RENDEMENT DE L'ÉDUCATION, SUR UN ECHANTILLON D'HOMMES}

Un problème bien connu lorsque l'on étudie le salaire des femmes est que nombreuses sont celles qui cessent de travailler pendant un certain temps. Leur participation au marché du travail est alors sélective ce qui génère un biais sur leurs salaires, biais mis en évidence par Gronau (1973) et Heckman (1974). De plus, en vertu de la théorie de la discrimination, les employeurs offriraient aux femmes des rémunérations plus faibles qu'aux hommes, à productivité égale.

De la même manière que Card and Krueger (1992), Goux et Maurin (1994) et Grogger (1996) notamment, nous choisissons donc de focaliser sur les hommes uniquement, notre étude des taux de rendement de l'éducation.

12 En réalité, l'estimation est compliquée par le fait que les écarts-types produits par la seconde étape des DMC sont biaisés. La plupart des logiciels de traitement statistique intègrent les corrections nécessaires. Nous utilisons pour notre part, la procédure SYSLIN de SAS. 


\subsection{Les variables retenues}

Définissons tout d'abord les variables dépendantes des équations (8), (9) et (10).

$\mathrm{PART}_{\mathrm{i}}=1$ si l'individu a perçu un revenu non nul au cours du mois dernier et $\mathrm{PART}_{\mathrm{i}}=0$ sinon.

$\mathrm{S}_{\mathrm{i}}=$ le niveau d'éducation, c'est-à-dire le nombre d'années d'études réussies,

$Y_{i}=$ les revenus perçus par l'individu i. Comme Trejo (1997), nous étudions le taux de salaire horaire ou plus précisément, le taux de revenu horaire puisque notre échantillon ne se limite pas aux seuls salariés.

Définissons ensuite les variables explicatives :

$\mathrm{T}_{\mathrm{i}}=$ le type d'éducation, public ou privé. Il s'agit d'une variable indicatrice qui prend la valeur 1 quand l'individu a été inscrit dans un plus grand nombre d'écoles publiques que d'écoles privées au cours de sa scolarité, et la valeur $0 \operatorname{sinon}^{13}$. Bien qu'impure, cette définition du type d'éducation reçue nous permet de disposer d'un nombre d'observations par catégorie plus élevé que si nous n'avions conservé que les individus ayant reçu une éducation totalement publique (45\% de la population des non migrants âgés d'au moins 18 ans) ou totalement privée (23\%).

13 Comme nous ne connaissons pas le nombre d'années passées au sein de chacun de ces établissements, nous nous référons au nombre d'établissements fréquentés dans le public et dans le privé. Nous faisons alors l'hypothèse qu'un individu ayant été inscrit dans un plus grand nombre d'écoles publiques que d'écoles privées au cours de sa scolarité, a reçu une éducation majoritairement publique. 
Les autres variables explicatives sont présentées dans les vecteurs ci-dessous.

$$
Z_{3 i}=\left(\begin{array}{l}
T \\
A G E * T \\
A G E 2 * T \\
A G E \\
A G E 2 \\
\text { REVPAR } \\
\text { RELIG } \\
\text { REDTOT } \\
\text { NIVMER } \\
\text { MICROPER } \\
\text { MICROMER } \\
\text { UNION } \\
\text { PRESENF 6 } \\
\text { FORMEL1 }
\end{array}\right) \quad\left(\begin{array}{l}
T \\
A G E * T \\
A G E 2 * T \\
A G E \\
A G E 2 \\
\text { REVPAR } \\
\text { RELIG } \\
\text { NIVPER } \\
\text { NIVMER }
\end{array}\right) \quad Z_{1 i}=Z_{2 i}=\left(\begin{array}{l}
A G E \\
A G E 2 \\
\text { NIVPER } \\
\text { NIVMER }
\end{array}\right)
$$

En fait, la difficulté principale dans la mise en œuvre de la procédure d'instrumentation réside dans le choix des instruments, c'est-à-dire des variables qui vont permettre de procéder à l'estimation de $S_{i}$ et de $S_{i}{ }^{2}$. En effet, parmi les déterminants acceptables de $S_{i}$ et de $S_{i}{ }^{2}$, on peut compter sur les variables explicatives non endogènes figurant dans la régression de base $\left(\mathrm{X}_{\mathrm{i}}\right.$ et $\mathrm{T}_{\mathrm{i}}$ ). Mais d'autres instruments sont nécessaires pour permettre l'identification de tous les paramètres. Ils doivent alors être très corrélés avec $S_{\mathrm{i}}$ et $\mathrm{S}_{\mathrm{i}}^{2}$ mais nullement avec les résidus de $\ln Y_{\mathrm{i}}$.

En tant que variables instrumentales, nous retenons quatre variables dont Arestoff et Bommier (2000) ont montré l'impact sur la réussite scolaire ${ }^{14}$. Aucun problème d'identification ne se pose puisque nous instrumentons deux variables à l'aide de quatre instruments. Notre modèle demeure donc suridentifié. Ces variables sont les suivantes :

$\left(\mathrm{AGE}^{*} \mathrm{~T}\right)=($ âge $*$ type d'éducation $)$

et $(\mathrm{AGE} 2 * \mathrm{~T})=\left[(\hat{a g e})^{2} *\right.$ type d'éducation $]$, qui rendent compte de l'évolution dans le temps de l'impact du type d'éducation sur le niveau des études,

REVPAR, le revenu des parents quand l'individu avait 15 ans, et RELIG, la religion de l'individu.

\footnotetext{
14 Les variables explicatives de $\mathrm{S}_{1}$ et $\mathrm{S}_{\mathrm{i}}^{2}$ sont les mêmes donc les vecteurs $\mathrm{Z}_{1 \mathrm{i}}$ et $\mathrm{Z}_{2 \mathrm{i}}$ sont confondus.
} 
Etant donné que nous considérons le niveau d'études comme un des déterminants de la participation au marché du travail, nous incluons les instruments de $\mathrm{S}$ (et de $\mathrm{S}_{\mathrm{i}}^{2}$ ) dans le vecteur $Z_{3 i}$ en lieu et place de la variable $S_{i}$ elle-même. Nous souhaitons que le coefficient de sélection $(\lambda)$ qui va résulter de l'estimation de la participation au marché du travail constitue une variable exogène dans l'équation de gains. Il est donc nécessaire qu'il ne dépende pas du niveau d'études non instrumenté.

Hormis les instruments de $S_{\mathrm{i}}$ et de $S_{\mathrm{i}}^{2}$, le vecteur $Z_{3 \mathrm{i}}$ comprend d'autres variables explicatives que l'on retrouve, pour certaines d'entre elles, dans $Z_{1 i}, Z_{2 i}$ et dans $X_{i}$ :

AGE et AGE2. Il s'agit de l'âge et de son carré.

On s'attend à ce que la propension à participer au marché du travail augmente jusqu'à un certain âge (signe positif de la variable AGE) puis diminue aux âges les plus avancés (signe négatif de AGE2)

Dans l'équation de gains, l'âge est utilisé comme une proxy de l'expérience professionnelle. En effet, l'information disponible est insuffisante pour déterminer précisément cette dernière. L'enquête renseigne sur le temps écoulé depuis le premier emploi mais il s'agit du premier emploi de plus de 6 mois ce qui occulte les emplois de moindre durée pendant lesquelles l'individu a néanmoins pu accumuler de l'expérience. En contrepartie, on aurait pu assimiler la totalité du temps écoulé depuis la fin des études à de l'expérience. Cette «expérience potentielle », selon les termes de Mincer, est la plus souvent retenue dans les études en coupe instantanée mais elle tend à surestimer l'expérience effective. En effet, elle ne prend en compte ni les interruptions de travail pour cause de chômage, ni les formations post-scolaires (Baker et al., 1995) ${ }^{15}$. Elle présente donc un caractère endogène car à âge et diplômes égaux, la durée de l'expérience professionnelle n'est pas la même pour tous.

Comme Sahn and Alderman (1988), Angrist and Krueger (1991), Card and Krueger (1992), Angrist (1995), Angrist and Lavy (1997) et Harmon and Walker (1999), nous substituons donc l'âge de l'individu, totalement exogène, à son expérience.

REDTOT $=$ le nombre total de redoublements de l'individu au cours de sa scolarité. Nous supposons que les redoublements peuvent être déterminants dans la participation au marché du travail car ils fournissent à l'employeur un indicateur des compétences du postulant à l'emploi. En revanche, nous excluons la possibilité qu'après un certain nombre d'années d'expérience professionnelle, les redoublements puissent encore influencer le revenu perçu.

\footnotetext{
15 Pour les femmes, cette surestimation de l'expérience est exacerbée car leurs interruptions de travail sont plus
} fréquentes (grossesse, etc). 
NIVPER et NIVMER, soient les nombres d'années d'études réussies du père et de la mère.

MICROPER et MICROMER désignent les secteurs d'activité du père et de la mère quand l'individu avait 15 ans. Ces deux variables prennent la valeur 1 lorsque les parents étaient micro-entrepreneurs ou travailleurs à leur propre compte, et la valeur 0 sinon. Un individu, dont le père travaille (ou a travaillé) dans le secteur informel, a toujours la possibilité de travailler avec lui (ou avec ses relations), accroissant de ce fait sa probabilité de participer au marché du travail.

UNION, variable dichotomique qui traduit la situation maritale. On la retrouve notamment chez Sahn and Alderman (1988), Angrist and Krueger (1991) et Glewwe (1996). Elle vaut 1 si l'individu est marié ou vit en union libre (ce qui est le cas de $61,8 \%$ de la population de notre échantillon), et 0 s'il est veuf, célibataire ou divorcé (séparé).

PRESENF6 = Présence d'enfants de moins de six ans dans le ménage. Il s'agit d'une variable dichotomique valant 1 si le ménage comprend des enfants de moins de six ans et 0 sinon. A Antananarivo, 69,5\% des individus étudiés font partie de ménages dans lesquels il n'y a pas de jeunes enfants (PRESENF6 $=0$ ).

Selon Sahn and Alderman (1988), UNION et PRESENF6 peuvent influer sur la décision de participer au marché du travail en raison de leur effet sur le salaire de réservation. Le mariage et les enfants à élever augmenteraient la probabilité de travailler des hommes mais diminueraient celle des femmes.

FORMEL1. Cette variable vaut 1 si l'individu exerce ou a exercé son premier emploi de plus de six mois dans le secteur formel (secteur public ou secteur privé formel). Elle vaut 0 si l'individu n'a jamais exercé un emploi pendant plus de six mois ou si ce premier emploi appartenait au secteur informel. On s'attend à ce qu'un premier emploi dans le secteur formel facilite ensuite la participation au marché du travail.

Signalons enfin que contrairement à Boumahdi et Plassard (1992), nous renonçons aux caractéristiques de l'emploi et de l'entreprise comme variables explicatives du revenu. En effet, en raison de leur simultanéité avec le revenu, ces variables ont tout lieu d'être endogènes.

\subsection{Estimation de l'équation de Mincer et de ses premières extensions}

Trois modèles sont présentés dans le tableau 3.

Le modèle (a) reprend l'équation standard de Mincer estimée par les MCO. Elle est enrichie dans le modèle (b) grâce à l'ajout du niveau d'éducation au carré et de variables caractérisant 
l'environnement familial. Enfin, nous appliquons la procédure d'Heckman (1979) afin de contrôler le biais d'auto-sélection de la force de travail. L'estimation de la participation au marché du travail par un modèle Probit est présentée en annexe 1 tandis que le modèle de gains, dont les variables explicatives comprennent désormais l'inverse du ratio de Mills $(\lambda)$, correspond au modèle (c).

Tableau 3 : Equations de revenus des hommes, en 1997

\begin{tabular}{|c|c|c|c|}
\hline Variable dépendante $: \ln Y$ & $\begin{array}{c}\begin{array}{c}\text { Equation standard } \\
\text { de Mincer (MCO) } \\
\text { (modèle a) }\end{array} \\
\end{array}$ & $\begin{array}{c}\text { Equation étendue } \\
\text { de Mincer (MCO) } \\
\text { (modèle b) }\end{array}$ & $\begin{array}{c}\text { Modèle (b) corrigé du } \\
\text { biais de sélection } \\
\text { (modèle } c)\end{array}$ \\
\hline Constante & $-1,67^{* * *}(0,22)$ & $-1,67^{* * * *}(0,24)$ & $-1,14^{* * *}(0,3)$ \\
\hline Niveau d'études & $0,1^{* * *}(0,007)$ & $0,02(0,03)$ & $0,02(0,03)$ \\
\hline (Niveau d'études) $^{2}$ & & $0,003^{* * *}(0,001)$ & $0,003^{* *}(0,001)$ \\
\hline Age & $0,02^{* *}(0,01)$ & $0,03^{* * *}(0,01)$ & $0,003(0,01)$ \\
\hline$(\text { Age })^{2}$ & $-0,0001(0,0001)$ & $-0,0001(0,0001)$ & $0,0002(0,0002)$ \\
\hline Niveau d'études du père & & $0,03^{* *}(0,01)$ & $0,03^{* *}(0,01)$ \\
\hline Niveau d'études de la mère & & $0,02(0,02)$ & $0,03^{*}(0,02)$ \\
\hline $\begin{array}{l}\text { Coefficient de sélection lié à la } \\
\text { participation au marché du travail }(\lambda)\end{array}$ & & & $-0,31^{* *}(0,15)$ \\
\hline $\mathrm{R}^{2}$ corrigé $($ en $\%)$ & 27 & 29 & 29 \\
\hline Nombre d'observations & 639 & 636 & 629 \\
\hline
\end{tabular}

Source : Enquête SET97, enquête 1-2-3 phase1 (1997), nos propres calculs.

Seuil de significativité : $* * * 1 \%, * * 5 \%, * 10 \%$. Les écarts-types sont notés entre parenthèses.

Le passage du modèle (a) aux modèles (b) et (c) met en évidence une notable variation du taux de rendement de l'éducation et de sa significativité.

Dans l'équation standard de Mincer [modèle (a)], le taux de rendement de l'éducation atteint $10 \%$ ce qui signifie qu'une année d'études supplémentaire génère un accroissement du logarithme du revenu horaire de $10 \%$. Il se révèle alors plus faible que celui de $13,4 \%$ que Psacharopoulos (1994) calcule, en moyenne, pour l'Afrique, entre 1970 et 1990 environ.

Lorsque l'éducation prend une forme non plus linéaire mais quadratique et que le niveau d'éducation des parents, supposé représenter l'environnement familial, est introduit dans l'équation de Mincer, le taux de rendement de l'éducation chute [modèle (b)]. Sachant que le niveau d'éducation moyen de notre échantillon est de 7,9 ans, une année d'éducation supplémentaire n'apporte plus qu'un surcroît de rémunération de $6,7 \%$. Il convient cependant de relativiser ce chiffre dans la mesure où seul le carré de l'éducation est significatif ${ }^{16}$.

Si l'on avait conservé un effet linéaire de l'éducation sur les revenus dans un modèle similaire au modèle (b), le taux de rendement de l'éducation obtenu aurait été de 8\%, significatif au seuil de $1 \%$. La prise en compte de l'environnement parental conduit donc, à elle seule, à une diminution du taux de rendement de deux points de pourcentage.

16 La significativité de $\mathrm{S}^{2}$ et la non-significativité de $\mathrm{S}$ confirment l'existence de taux de rendement hétérogènes postulés par Card (1998). 
D'après l'estimation du modèle (c), la sélection des individus sur le marché du travail est effectivement non aléatoire. Le signe négatif et significatif de la variable $\lambda$ montre que les caractéristiques non observées qui tendent à favoriser la participation au marché du travail affectent également le revenu potentiel en contribuant à le réduire. Contrairement au résultat attendu, les hommes qui ne travaillent pas, soient ceux pour lesquels on ne connaît pas $\operatorname{lnY}$, se verraient donc offrir des revenus supérieurs à ceux des hommes inclus dans l'échantillon. Cela pourrait signifier que l'accès à certains emplois est rendu difficile par l'existence de barrières à l'entrée qui obligent les individus désirant travailler à accepter des emplois plus faiblement rémunérés ${ }^{17}$.

Quelle que soit l'ampleur du biais de sélection, son influence sur le taux de rendement de l'éducation semble négligeable puisque celui-ci ne se trouve nullement modifié du modèle (b) au modèle (c). Les signes conjointement positifs du niveau d'études (qui demeure non significatif) et de son carré viennent démontrer l'existence de rendements croissants de l'éducation à Madagascar, ce qui confirme les anticipations de l'analyse descriptive, et plus précisément du tableau 1.

\subsection{Instrumentation du niveau d'études et intégration du type d'éducation dans l'équation de Mincer}

Nous cherchons d'abord à améliorer le modèle (c) en ajoutant le type d'éducation (public ou privé) au modèle (c) et à l'équation de participation qui lui est associée. Il apparaît qu'avoir reçu une éducation publique plutôt que privée n'influe significativement ni sur la probabilité de travailler ni sur le revenu horaire. Les variables explicatives du modèle (c) conservent leur significativité ou leur non-significativité. Le taux de rendement de l'éducation connaît une légère augmentation, passant de $6,7 \%$ à $7,7 \%$.

Il est cependant trop tôt encore pour affirmer d'une part que le taux de rendement de l'éducation se fixe à $8 \%$ à Antananarivo, et d'autre part que le caractère public ou privé de l'éducation reçue n'exerce aucun impact sur le revenu horaire d'un travailleur, en 1997. En effet, nous avons préalablement démontré qu'il était nécessaire d'instrumenter le niveau

17 Tous les individus «employables » ne souhaitent cependant pas travailler. Ainsi, la probabilité de participer au marché du travail, associée au modèle (c) et présentée en annexe 1, apparaît comme étant négativement reliée au niveau d'éducation de l'individu lui-même et de ses parents (seul le niveau d'éducation de la mère est significatif). Ce phénomène peut s'interpréter par le fait que l'éducation, en augmentant le salaire de réservation, réduit la participation au marché du travail. Le chômage d'attente à l'entrée dans le secteur formel semble, en effet, relativement qualifié à Antananarivo : 62,8\% des chômeurs sont allés au-delà du cycle d'études primaires et 12,8\% se sont inscrits à l'université (Projet MADIO, 1997). 
d'études pour améliorer l'estimation du taux de rendement de l'éducation. Dans cette optique, le graphique 1 offre un rappel de la procédure suivie pour estimer le revenu horaire.

Graphique 1 : Synthèse de la procédure d'estimation des revenus

Correction du biais de sélection :

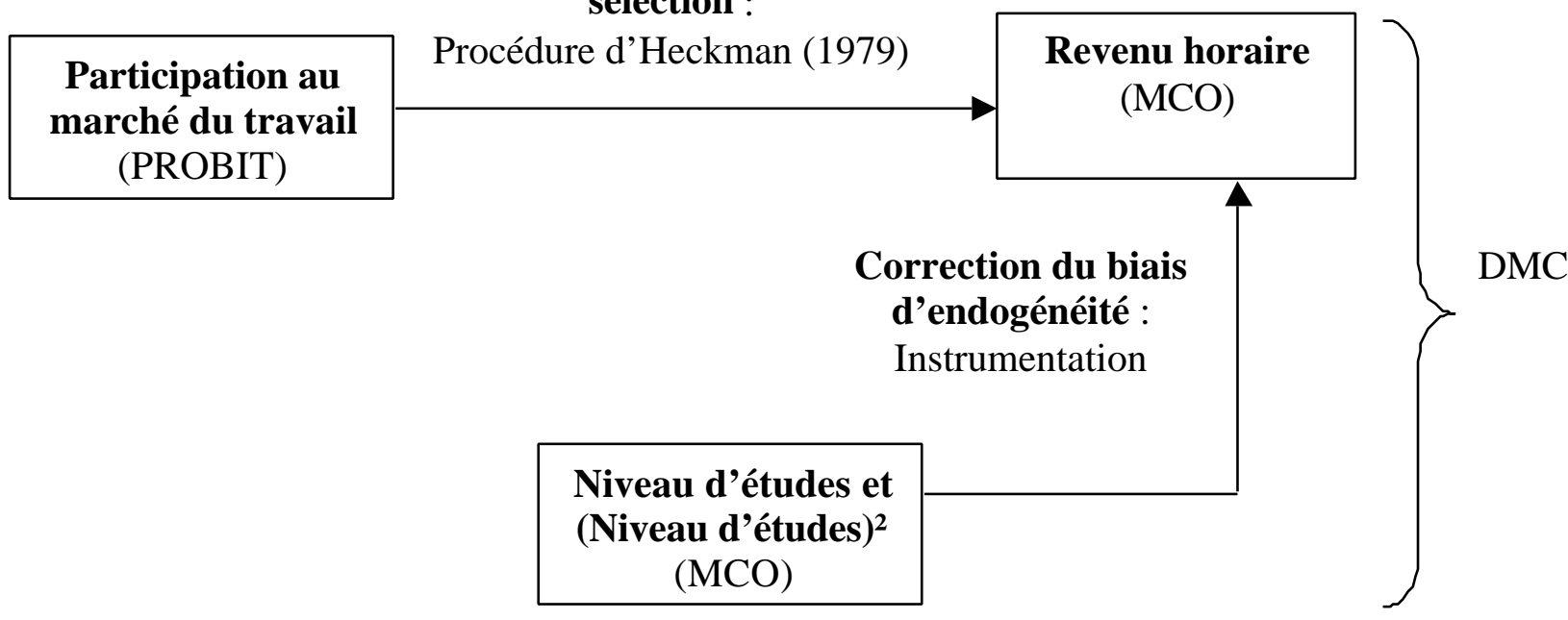

\subsubsection{Validité de l'instrumentation}

Le tableau 4 fournit les résultats des estimations de la participation au marché du travail, du niveau d'études et enfin du taux de revenu horaire, soient les modèles (d), (e) et (f), respectivement. Les résultats de l'instrumentation de la variable (Niveau d'études) ${ }^{2}$ sont présentés en annexe 1.

Il convient de commencer par vérifier la qualité des instruments retenus. La méthode consiste à régresser les résidus des DMC [modèle (f)] sur les instruments ne figurant pas parmi les variables explicatives de $\ln Y_{i}$ [(Greene, 1997, p.288) et (Maddala, 1992, p.366)].

Nos instruments, soient le revenu des parents, la religion et les variables croisant l'âge et le type d'éducation, s'avèrent alors parfaitement valides. 
Tableau 4 : Modèle de gains corrigé du biais de sélectivité, avec niveau d'études instrumentés, pour les hommes, en 1997

\begin{tabular}{|c|c|c|c|}
\hline & $\begin{array}{l}\text { Participation au } \\
\text { marché du travail } \\
\text { (Probit) } \\
\text { (modèle d) }\end{array}$ & $\begin{array}{l}\text { Niveau d'études : } \\
\text { instrumentation } \\
(\text { MCO) } \\
\text { (modèle e) }\end{array}$ & $\begin{array}{c}\text { Equation de } \\
\text { revenus : } \\
\text { estimation de } \ln Y \\
\text { (DMC) } \\
\text { (modèle } f \text { ) } \\
\end{array}$ \\
\hline Constante & $-0,4(0,59)$ & $-2,66^{*}(1,37)$ & \\
\hline $\begin{array}{l}\text { Niveau d'études instrumenté } \\
\text { (Niveau d'études)² instrumenté }\end{array}$ & & & $\begin{array}{c}0,16(0,17) \\
-0,007(0,009)\end{array}$ \\
\hline Age & $0,07^{* *}(0,03)$ & $0,27^{* * *}(0,06)$ & $0,02(0,01)$ \\
\hline$(\text { Age })^{2}$ & $-0,0009^{* * *}(0,0003)$ & $-0,003^{* * *}(0,0007)$ & $-0,00002(0,0002)$ \\
\hline \multicolumn{4}{|l|}{ Type d'éducation } \\
\hline Publique & $-2,45^{* * *}(0,8)$ & $-5,14^{* *}(2,17)$ & $0,04(0,09)$ \\
\hline Privée & Ref & Ref & Ref \\
\hline Age*Type d'éducation & $0,15^{* * *}(0,04)$ & $0,29^{* * *}(0,11)$ & \\
\hline (Age) ${ }^{2 * T y p e ~ d ' e ́ d u c a t i o n ~}$ & $-0,002^{* * *}(0,0005)$ & $-0,004^{* * *}(0,001)$ & \\
\hline Revenu des parents & $-0,0005(0,0006)$ & $0,005^{* * *}(0,002)$ & \\
\hline \multicolumn{4}{|l|}{ Religion } \\
\hline Catholique & $0,39^{* * *}(0,13)$ & $-0,12(0,28)$ & \\
\hline Autre religion & Ref & Ref & \\
\hline Nombre de redoublements & $-0,15^{* * *}(0,05)$ & & \\
\hline Niveau d'études du père & $-0,03(0,02)$ & $0,37^{* * *}(0,06)$ & $0,07^{* * *}(0,01)$ \\
\hline Niveau d'études de la mère & $-0,06^{* *}(0,03)$ & $0,54^{* * *}(0,07)$ & $0,08^{* * *}(0,02)$ \\
\hline \multicolumn{4}{|l|}{ Activité professionnelle du père } \\
\hline Micro-entrepreneur ou à son propre compte & $0,03(0,14)$ & & \\
\hline Autre & Ref & & \\
\hline \multicolumn{4}{|l|}{ Activité professionnelle de la mère } \\
\hline Micro-entrepreneur ou à son propre compte & $-0,05(0,13)$ & & \\
\hline Autre & Ref & & \\
\hline \multicolumn{4}{|l|}{ Statut marital } \\
\hline Marié ou en union libre & $0,72^{* * *}(0,16)$ & & \\
\hline Autres situations & Ref & & \\
\hline \multicolumn{4}{|l|}{$\begin{array}{l}\text { Présence d'enfants de moins de } 6 \text { ans } \\
\text { dans le ménage }\end{array}$} \\
\hline Oui & $0,3^{*}(0,18)$ & & \\
\hline Non & Ref & & \\
\hline \multicolumn{4}{|l|}{ Type du premier emploi } \\
\hline Formel & $0,57^{* * * *}(0,12)$ & & \\
\hline Informel & Ref & & \\
\hline $\begin{array}{l}\text { Coefficient de sélection lié à la } \\
\text { participation au marché du travail }(\lambda)\end{array}$ & & & $-0,48^{* * *}(0,16)$ \\
\hline Log de la vraisemblance & 292,2 & & \\
\hline $\mathrm{R}^{2}$ corrigé (en $\%$ ) & & 37 & 18 \\
\hline Nombre d'observations & 813 & 636 & 629 \\
\hline
\end{tabular}

Source : Enquête SET97, enquête 1-2-3 phase1 (1997), nos propres calculs.

Note $:$ Ref $=$ variable indicatrice de référence.

Seuil de significativité : ***1\%,**5\%,*10\%. Les écarts-types sont notés entre parenthèses.

Parallèlement, il nous faut vérifier l'endogénéité du niveau d'éducation et de son carré, les variables que nous avons instrumentées. Un test naturel d'endogénéité est le test d'Hausman (1978). Il se fonde sur la comparaison des estimateurs des MCO et des DMC et vérifie s'ils sont ou non, significativement différents. En pratique, il suffit de régresser les résidus de l'instrumentation sur ceux des DMC, par des MCO [(Greene, 1997, p. 443) et (Maddala, 1992, p.506)]. 
Dans notre étude, l'endogénéité de $\mathrm{S}$ et celle de $\mathrm{S}^{2}$ sont vérifiées au seuil de $1 \%$. La méthode des DMC fournit donc des estimateurs convergents, au contraire des estimateurs issus de la méthode des MCO, qui sont biaisés et non convergents.

\subsubsection{Participation au marché du travail}

Le type d'éducation constitue un déterminant significatif de la probabilité de travailler. Toutes choses égales par ailleurs, les anciens élèves de l'enseignement public participent significativement moins au marché du travail que les anciens élèves de l'enseignement privé. Si l'on en juge par le signe des variables croisant l'âge et le type d'éducation, ce phénomène affecterait principalement les plus jeunes cohortes. Nous pouvons le vérifier en représentant graphiquement l'effet de chacun des deux types d'éducation en fonction de l'âge.

Effet total de l'éducation publique $=-2,45+(0,07$. Age $)-\left(0,0009\right.$. Age $\left.^{2}\right)+(0,15$. Age $)-$ $\left(0,002 . \mathrm{Age}^{2}\right)=-2,45+(0,22 . \mathrm{Age})+\left(0,0029 . \mathrm{Age}^{2}\right)$.

Effet total de l'éducation privée $=(0,07$. Age $)-\left(0,0009\right.$. Age $\left.^{2}\right)$.

Graphique 2 : Effet total du type d'éducation sur la participation au marché du travail des hommes, en 1997, en fonction de leur âge

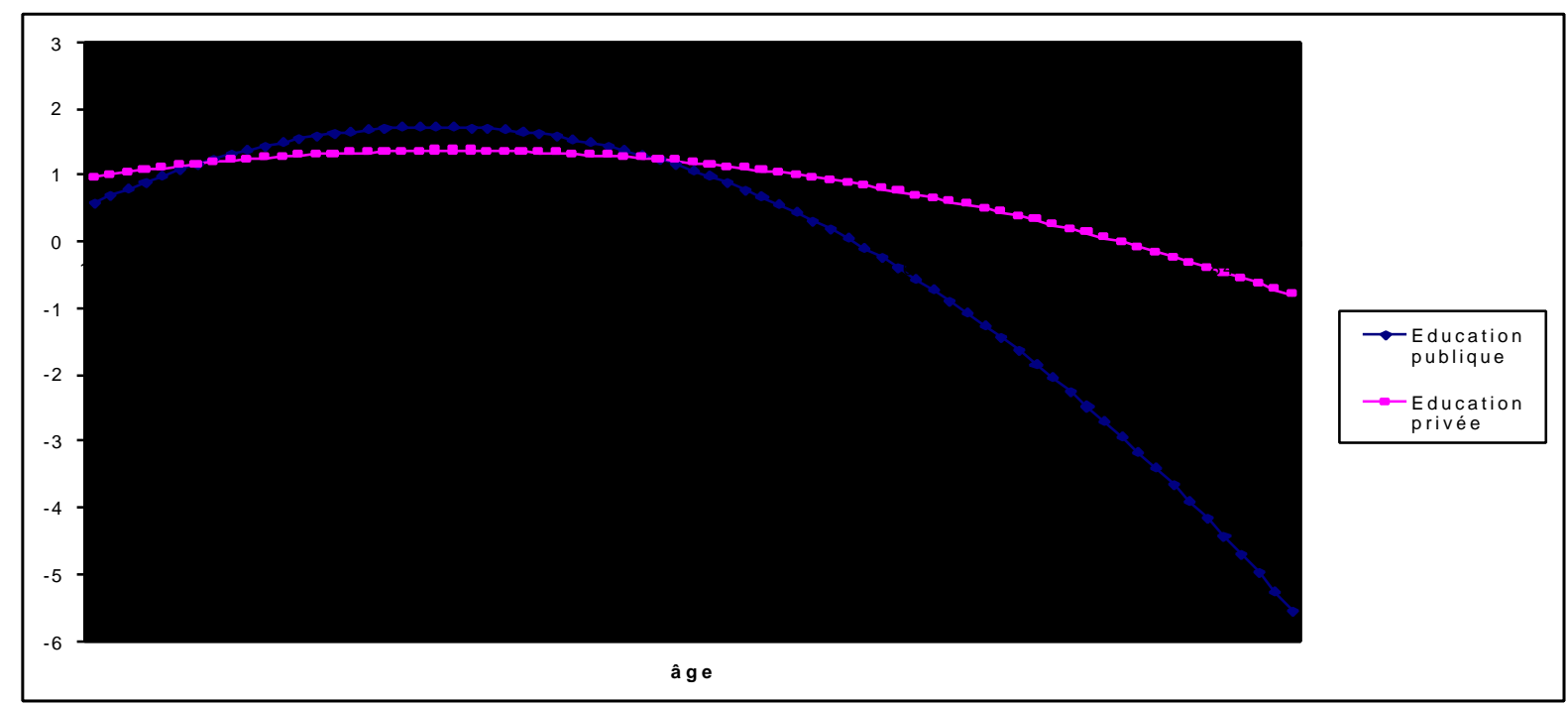

Source : Enquête SET97, enquête 1-2-3 phase1 (1997), nos propres calculs.

Le graphique 2 montre alors que ce sont les moins de 25 ans et les plus de 50 ans qui pâtissent plus spécifiquement d'une scolarisation publique plutôt que privée. Pour les jeunes générations, cet effet vient confirmer la dégradation de l'éducation publique constatée par Arestoff et Bommier (2000). 
Les autres variables explicatives exercent, dans leur ensemble, des effets classiques sur la participation au marché du travail.

Les signes positif de l'âge et négatif de son carré, tous deux significatifs, épousent donc le cycle de vie du travailleur.

Enfin le fait que la probabilité de travailler diminue avec l'éducation de la mère peut résulter de prétentions salariales trop élevées pour les individus issus de milieux favorisés.

\subsubsection{Revenus et taux de rendement de l'éducation}

L'instrumentation du niveau d'études provoque une notable perte de significativité du modèle de gains, le $\mathrm{R}^{2}$ corrigé passant de $29 \%$ dans le modèle (c) ${ }^{18}$ à $18 \%$ dans le modèle (f). Seuls le coefficient de sélection lié à la participation au marché du travail et le niveau d'études des parents semblent désormais influencer significativement les revenus.

On retrouve cet effet direct positif de l'éducation des parents sur le revenu de leur enfant dans Altonji and Dunn (1996), bien que leur étude porte sur les Etats Unis, mais aussi dans Heckman and Hotz (1986) au Panama. Comme ces derniers, nous constatons que le poids de l'éducation maternelle l'emporte sur celui de l'éducation paternelle. Selon Mincer (1993), le retrait maternel du marché du travail lié à la grossesse ou à l'éducation des enfants constitue, en effet, une composante de l'investissement parental dans le capital humain de leurs enfants car le coût d'opportunité, auquel la mère fait face, est d'autant plus élevé qu'elle est éduquée ${ }^{19}$.

Le type d'éducation demeure non significatif, avec ou sans instrumentation. Nous pouvons donc affirmer que l'éducation publique ou privée joue un rôle sur la participation au marché du travail mais pas sur la rémunération des travailleurs.

Le niveau d'études lui-même ne constitue plus un déterminant significatif du revenu horaire ${ }^{20}$. Les facteurs non observés telles que les capacités intrinsèques de chacun, que l'instrumentation permet de prendre en compte, capteraient donc la totalité de l'effet initialement supposé de l'éducation sur les revenus. Par conséquent, il semblerait que l'éducation n'exerce en elle-même aucun impact direct sur les revenus de l'individu. Elle ne servirait que d'indicateur de la motivation et des facilités de chacun à travailler.

\footnotetext{
18 et dans le modèle non représenté qui intègre le type d'éducation parmi les variables explicatives sans encore instrumenter le niveau d'éducation

19 De ce fait, on devrait prendre en compte une partie des gains de l'enfant dans la détermination du rendement de l'éducation de la mère.

20 Ce résultat perdure si l'on suppose un effet linéaire et non plus quadratique du niveau d'études sur les revenus.
} 
Bien qu'il s'agisse là d'une conclusion qui ne serait pas sans déplaire aux défenseurs de la théorie du filtre, elle nous semble peu satisfaisante. Nous approfondissons alors notre analyse en remettant en cause l'hypothèse d'un marché du travail concurrentiel. En effet, la taille et les caractéristiques du secteur informel à Antananarivo laissent plutôt supposer l'existence d'un marché du travail segmenté.

\section{TAUX DE RENDEMENT DE L'ÉDUCATION SOUS L'HYPOTHĖSE D'UNE SEGMENTATION DU MARCHÉ DU TRAVAIL}

Selon la théorie du dualisme, le marché du travail n'a rien de walrassien. Le fonctionnement des deux secteurs qui le composent et les caractéristiques de la force de travail que chacun d'eux mobilise ne résulteraient donc pas d'imperfections de la concurrence qui s'exercent sur ce marché pris comme un tout, mais de leur logique propre. Il devient donc nécessaire d'analyser les déterminants du revenu en distinguant celui-ci selon le secteur auquel il appartient.

\subsection{Théorie de la segmentation du marché du travail : une brève présentation $^{21}$}

La théorie de la segmentation du marché du travail connaît son essor pendant les années 70 grâce, notamment, aux travaux de Doeringer and Piore (1971) puis Piore (1978). Elle conteste l'unité de fonctionnement du marché du travail supposée par les néo-classiques et postule, au contraire, l'existence de divers espaces de mobilité au sein du marché du travail.

Dans la version dualiste, le marché du travail est composé de deux secteurs : le secteur primaire vers lequel tous les chômeurs sont attirés et le secteur secondaire. Le secteur primaire regroupe des entreprises ou segments d'entreprise organisés en marchés internes afin de stabiliser la main-d'œuvre. Les affectations, promotions et rémunérations de la maind'œuvre y sont régies selon des modalités particulières résultant largement de l'histoire des relations sociales et de considérations administratives, à l'intérieur de la firme et dans son environnement. Selon Piore and Doeringer (1971), ces marchés internes sont en grande partie déconnectés des confrontations «externes » entre offreurs et demandeurs de travail. Dans ces conditions, les différentiels de revenus entre les qualifiés et les moins qualifiés peuvent ne pas être nécessairement reliés aux différentiels de productivité. Le secteur secondaire fonctionne, en revanche, selon un mode plus concurrentiel.

\footnotetext{
21 Taubman and Wachter (1986) proposent une revue de la littérature exhaustive sur les marchés du travail segmentés.
} 
Ces secteurs ne correspondent donc ni aux mêmes types d'emplois, ni aux mêmes modes de fonctionnement. Les emplois du secteur primaire sont mieux rémunérés, plus qualifiés, bénéficient de conditions de travail et de perspectives de carrière plus favorables que ceux du secteur secondaire. Les travailleurs du secteur primaire sont plus anciens, mieux formés et mieux organisés collectivement que ceux du secteur secondaire. Or, en raison de barrières à l'entrée mais aussi de politiques d'embauche discriminatoires, il est difficile de passer du secteur secondaire au secteur primaire.

La théorie de la segmentation paraît transposable au cas des pays en développement où le marché du travail connaît globalement de nombreux dysfonctionnements. En effet, comme le secteur formel est incapable d'absorber toute la main-d'œuvre disponible, le secteur informel peut être considéré comme une «roue de secours » procurant emploi et revenu à ceux qui n'ont pu accéder au secteur formel (ou moderne), et notamment aux moins éduqués.

Coquery-Vidrovitch (1991) écrit, à propos du secteur informel dans les villes africaines (p.183) :

«Ce marché est irrégulier et terriblement vulnérable car non protégé et hautement concurrentiel; il est, en effet, infiniment plus aisé d'y rentrer que dans le secteur capitaliste».

Si l'on attribue les caractéristiques du secteur primaire au secteur moderne et celles du secteur secondaire, à l'informel, comme le fait Doeringer (1988) dans le cas de la Jamaïque, alors tout porte à croire qu'en 1997, le marché du travail à Antananarivo est segmenté. A titre d'illustration, Ramilison (1997) montre ainsi que la mobilité du secteur formel au secteur informel existe, alors qu'elle est faible dans l'autre sens.

\subsection{Revenus du travail dans les secteurs formel et informel : analyse descriptive}

Le secteur informel regroupe «l'ensemble des unités de production qui ne possèdent pas de numéro statistique ou, dans les cas des patrons et des travailleurs à leur propre compte, qui ne tiennent pas de comptabilité » (Projet MADIO, 1995).

En contrepartie, le secteur formel comprend l'ensemble des établissements publics (administration et entreprises), les établissements privés enregistrés à l'INSTAT (ou les travailleurs indépendants tenant une comptabilité), les entreprises associatives et les zones franches. 
Nous retenons donc une définition du secteur informel plus large que celle proposée par Roubaud (1994, p.23). En effet, ce dernier définit le secteur informel comme l'ensemble des industries microscopiques qui mobilisent de faibles quantités de facteurs de production (travail et capital), ne respectent pas la législation en vigueur, et génèrent un volume d'output infinitésimal à l'échelle des grands agrégats macro-économiques.

\subsubsection{Revenus horaires et niveau d'éducation, dans les secteurs formel et informel}

D'après le tableau 5, les individus ayant suivi des études primaires perçoivent, en moyenne, $74 \%$ de revenu horaire en plus dans le secteur formel, relativement au secteur informel. Mais cette supériorité des revenus dans le secteur formel ne tient pas pour les personnes très qualifiées. En effet, dans la population ayant accédé aux études supérieures, un léger avantage revient au secteur informel. Depuis les débuts de la crise économique et le tarissement du recrutement des fonctionnaires, les emplois informels seraient donc devenus plus lucratifs que les emplois formels qui sont proposés aux diplômés.

Tableau 5 : Revenu horaire moyen et niveau d'études, en 1997, dans les secteurs formel et informel

\begin{tabular}{ccccc}
\hline \hline en Fmg & $\begin{array}{c}\text { Sans éducation ou } \\
\text { Primaire }\end{array}$ & Secondaire 1 ${ }^{\text {er }}$ cycle & Secondaire 2nd cycle & Supérieur \\
\hline Secteur formel & 1101 & 1053 & 1370 & 2277 \\
Secteur informel & 632 & 658 & 847 & 2530 \\
\hline Sources:
\end{tabular}

Sources : Enquête SET97, enquête 1-2-3 phase1 (1997), nos propres calculs.

En termes de rendement supposé du niveau d'éducation, le tableau 5 met en évidence un faible accroissement (voire une diminution, dans le secteur formel) des bénéfices nets de la scolarisation lorsque les individus de niveau primaire accèdent au $1^{\text {er }}$ cycle du secondaire. Mais ce rendement croît par la suite. Il atteint respectivement $66 \%$ et $198 \%$ dans les secteurs formel et informel lors du passage du $2^{\text {nd }}$ cycle du secondaire au supérieur.

\subsubsection{Revenus horaires et type d'éducation, dans les secteurs formel et informel}

Le tableau 6 montre qu'il existe une relative homogénéité entre les revenus horaires moyens des anciens élèves de l'éducation publique et de l'éducation privée, pour un niveau d'études donné.

Néanmoins, on constate que pour les individus les moins éduqués (niveau primaire), ceux qui travaillent dans le secteur formel gagnent légèrement plus dès lors qu'ils ont reçu une éducation publique alors que ceux qui travaillent dans l'informel seront légèrement favorisés 
si leur éducation était privée. Pour des niveaux d'études plus élevés, le phénomène inverse se produit.

Tableau 6 : Revenu horaire moyen et type d'éducation, par niveau d'études, en 1997, dans les secteurs formel et informel

\begin{tabular}{ccccccc}
\hline \hline & \multicolumn{2}{c}{ Primaire } & \multicolumn{2}{c}{ Secondaire 1 ${ }^{\text {er }}$ cycle } & \multicolumn{2}{c}{ Secondaire 2nd cycle ou Supérieur $^{\text {en Fmg }}$} \\
\cline { 2 - 7 } & Public & Privé & Public & Privé & Public & Privé \\
\hline Secteur formel & 1154 & 1033 & 1009 & 1187 & 1684 & 1888 \\
Secteur informel & 638 & 684 & 703 & 615 & 1378 & 1375 \\
\hline
\end{tabular}

Sources : Enquête SET97, enquête 1-2-3 phase1 (1997), nos propres calculs.

Le type d'éducation exercerait donc une influence différenciée sur la rémunération des actifs selon que le secteur d'activité est formel ou informel. Toutefois, les écarts d'un type d'éducation à l'autre étant de faible ampleur, rien ne nous laisse supposer qu'ils seront significatifs au sein de chacun des secteurs d'activité.

\subsection{Taux de rendement de l'éducation dans les secteurs formel et informel}

Pour déterminer le taux de rendement de l'éducation dans un cadre de segmentation potentielle du marché du travail, il est nécessaire d'estimer un modèle de gains par secteur. En dépit des différences entre les deux secteurs, la liaison formation-productivité-revenus, soit le fondement de l'analyse orthodoxe du capital humain, n'est pas fondamentalement remise en cause au sein de chaque secteur. Dans le secteur primaire ou formel, on considérera simplement que l'éducation agit principalement sous sa forme spécifique, soit au travers de l'expérience professionnelle.

Le modèle de gains que nous retenons est proche du modèle (f), à trois exceptions près.

D'abord, le coefficient de sélectivité diffère : il est désormais lié, non plus à la participation au marché du travail, mais à l'affectation au secteur formel ou informel.

Pour corriger le biais de sélection sur le marché du travail (y participer ou pas) puis le biais d'affectation à l'un ou l'autre secteur d'activité (formel ou informel), nous estimons donc un modèle séquentiel en trois phases.

Ensuite, afin de disposer d'un échantillon de taille suffisante au sein de chacun des deux secteurs d'activité, nous ne nous limitons plus aux hommes et ajoutons une variable indicatrice du sexe parmi l'ensemble des régresseurs. En raison de la taille de notre 
échantillon, nous sommes donc contraints de remettre en cause les arguments préalablement développés en faveur d'une estimation des revenus portant sur les hommes uniquement. Nous pensons cependant que ce choix est préférable à celui qui consisterait à décomposer l'échantillon par secteur et par sexe, ce qui conduirait à l'obtention de résultats incohérents et non représentatifs. Certains auteurs ont, de surcroît, adopté la même démarche que la nôtre. C'est le cas de Glewwe (1996) qui étudie aussi les revenus par secteur d'activité dans un pays en développement, le Ghana.

Enfin, nous renonçons à la forme quadratique du niveau d'éducation car le modèle (f) a permis de montrer qu'une fois instrumenté, le niveau d'éducation au carré n'améliorait pas significativement le modèle.

\subsubsection{Affectation dans le secteur formel}

Les estimations de la participation au marché du travail et de l'affectation dans le secteur formel sont présentées en annexe 2. Elles permettent de calculer les termes de redressement de biais correspondants.

La probabilité de travailler dans le secteur formel s'avère significativement plus forte pour les hommes, pour les individus dont les parents sont éduqués, et pour ceux qui ont entamé leur carrière professionnelle au sein même du secteur formel.

Le coefficient positif et significatif de la variable âge confirme que les jeunes générations disposent d'une probabilité plus faible que leurs aînés d'intégrer le secteur formel, à niveau d'études et autres caractéristiques socio-économiques semblables.

En revanche, avoir reçu une éducation publique ou privée ne modifie pas de façon significative la probabilité de travailler dans le secteur formel. Les instruments du niveau d'études n'influencent d'ailleurs pas non plus significativement le secteur d'affectation.

\subsubsection{Taux de rendement de l'éducation}

Le tableau 7 présente l'estimation des revenus par secteurs. Il fait apparaître un taux de rendement de l'éducation qui atteint $7 \%$ dans le secteur informel mais qui s'avère non significatif dans le secteur formel.

Lorsque l'on compare ce taux de rendement avec celui qui résulte de l'estimation d'une équation standard de Mincer par secteurs (annexe 3), une nette divergence apparaît. 
Dans le secteur formel, le taux de rendement de l'éducation, de 9\% dans l'estimation mincerienne, perd sa significativité quand le niveau d'études est instrumenté, que les biais de sélection et d'affectation sont contrôlés, et que le type d'éducation et l'environnement parental deviennent des variables explicatives. La nécessité d'instrumenter le niveau d'éducation n'est pas remise en cause puisque d'après le test d'Hausman (1978), cette variable est endogène dans le secteur formel ${ }^{22}$.

Plusieurs raisons peuvent alors être invoquées pour expliquer cette absence de significativité du résultat.

Le rendement économique de l'éducation demeure fortement soumis au nombre d'éduqués qui offrent leur force de travail au cours de l'année étudiée. Angrist (1995) l'a vérifié dans le cas de la Palestine. A Antananarivo, en 1997, plus des trois quarts des salariés du secteur formel ont un niveau d'études secondaires ou supérieures. A environnement socioéconomique et expérience professionnelle donnés, le niveau d'études perd alors son caractère potentiellement explicatif des revenus.

Parallèlement, la non significativité du niveau d'études dans le secteur formel peut aussi résulter de l'existence d'un stock de travailleurs qualifiés excédentaire par rapport aux opportunités d'emplois qualifiés. Un processus de filtering down s'est mis en place dans la capitale malgache où des postes peu rémunérateurs attirent désormais des individus surqualifiés relativement aux compétences que ces emplois exigent (Coury, 2000). Au Kenya et en Tanzanie, Knight and Sabot (1987) ont constaté un phénomène du même type.

Tableau 7 : Estimation des revenus dans les secteurs formel et informel, en 1997

\begin{tabular}{|c|c|c|}
\hline & $\begin{array}{c}\text { InY dans le secteur formel } \\
\text { (DMC) }\end{array}$ & $\begin{array}{l}\text { InY dans le secteur informel } \\
\text { (DMC) }\end{array}$ \\
\hline Constante & $-1,95^{* * *}(0,42)$ & $-1,78^{* * *}(0,28)$ \\
\hline Niveau d'études instrumenté & $0,03(0,02)$ & $0,07^{* *}(0,17)$ \\
\hline Age & $0,04^{* * *}(0,01)$ & $0,03^{* * *}(0,01)$ \\
\hline$(\text { Age })^{2}$ & $-0,0002(0,0001)$ & $-0,0002 *(0,0001)$ \\
\hline \multicolumn{3}{|l|}{ Type d'éducation } \\
\hline Publique & $0,05(0,06)$ & $0,13^{*}(0,07)$ \\
\hline Privée & Ref & Ref \\
\hline Niveau d'études du père & $0,04^{* * *}(0,01)$ & $0,05^{* * *}(0,01)$ \\
\hline Niveau d'études de la mère & $0,06^{* * *}(0,01)$ & $0,05^{* * *}(0,02)$ \\
\hline \multicolumn{3}{|l|}{ Sexe } \\
\hline Homme & Ref & Ref \\
\hline Femme & $-0,11^{*}(0,06)$ & $-0,32^{* * * *}(0,07)$ \\
\hline Coefficient d'affectation sectorielle $\left(\lambda_{2}\right)$ & $-0,05(0,13)$ & $-0,33^{* *}(0,16)$ \\
\hline $\mathrm{R}^{2}$ corrigé (en \%) & 22,1 & 9,2 \\
\hline Nombre d'observations & 530 & 579 \\
\hline
\end{tabular}

Source : Enquête SET97, enquête 1-2-3 phase1 (1997), nos propres calculs.

Note $:$ Ref $=$ variable indicatrice de référence.

Seuil de significativité : ***1\%,**5\%,*10\%. Les écarts-types sont notés entre parenthèses.

22 Lorsque nous testons la qualité des instruments choisis, les variables croisant l'âge et le type d'éducation apparaissent comme étant de mauvais instruments : les résidus de l'estimation de $\ln Y$ dépendent significativement de ces variables. Le nombre d'instruments demeure toutefois suffisant pour que le modèle soit identifié. 
Dans le secteur informel, au contraire, les enrichissements du modèle de Mincer que nous proposons portent peu leurs fruits. Le taux de rendement demeure significatif et chute de moins d'un point de pourcentage, de 7,6\% exactement à 7\%. De fait, le test d'Hausman (1978) révèle l'exogénéité de la variable d'éducation. Une année d'études supplémentaire assure donc à un travailleur du secteur informel une augmentation de son taux de revenu horaire d'environ $7 \%$.

\subsubsection{Effet du type d'éducation et des autres variables}

Le type d'éducation n'est pas significatif dans le secteur formel. En revanche, il influence significativement les revenus perçus dans le secteur informel. Son coefficient positif indique alors que les employés de ce secteur reçoivent une rémunération horaire relativement plus élevée s'ils ont été éduqués dans des établissements publics plutôt que privés.

Pour justifier pourquoi il est préférable, lorsque que l'on travaille dans l'informel, d'avoir reçu une éducation publique, l'argument de l'homogénéité des individus ne tient pas. En effet, tant dans le formel que dans l'informel, la grande majorité des employés est issue de l'enseignement public. D'autres facteurs entrent donc en jeu que nous n'observons pas ici.

L'expérience professionnelle accroît les revenus de manière linéaire dans le secteur formel, à un taux d'environ 4\%. En revanche, dans le secteur informel, le profil expérience-gains présente une légère forme en cloche puisque le coefficient négatif de l'âge au carré est significatif. Bien qu'il ait aussi été vérifié au Costa Rica Gindling, 1991), ce résultat est contre-intuitif. En effet, comme le soulignent Smith and Metzger (1998) dans leur étude du secteur informel de Mexico, les faibles opportunités d'avancement dans le secteur informel laissent plutôt supposer un profil expérience-gains (ou âge-gains) relativement plat.

Malgré ces différences de profils, le poids de l'expérience dans la détermination des revenus demeure relativement plus important dans le secteur formel, ce qui peut résulter de la prise en compte des emplois publics (administration et entreprises publiques) dans les emplois formels. Les premiers résultats de l'enquête Emploi à Antananarivo (Projet MADIO, 1997) montrent ainsi que les emplois publics sont ceux pour lesquels l'ancienneté moyenne est la plus forte.

Toutes choses étant égales par ailleurs, l'impact positif du niveau d'études des parents sur les revenus individuels, de même que le préjudice dont sont victimes les femmes apparaît dans les deux secteurs. La discrimination sexuelle s'avère toutefois plus forte dans l'informel. 
Enfin, le signe négatif et significatif du coefficient d'affectation dans le secteur informel signifie que les individus les plus à même de travailler dans ce secteur sont aussi ceux qui gagneront le moins. Dans le secteur formel en revanche, le biais d'affectation sectorielle n'est pas significatif. Les caractéristiques non observées qui accroissent la probabilité d'exercer un emploi formel ne prédisposent donc pas les travailleurs concernés à gagner significativement plus ou moins que les autres. De fait, on peut penser que l'attirance pour le secteur formel ne résulte pas seulement du salaire escompté mais aussi de critères tels que la sécurité de l'emploi et les possibilités de promotion.

\subsubsection{Pertinence de l'hypothèse d'une segmentation du marché du travail : test de Chow et analyse de la variance.}

Pour valider les résultats du tableau 7, nous testons la stabilité des coefficients par l'analyse de la variance et le test de Chow. Nous cherchons ainsi à vérifier la pertinence de l'hypothèse d'un marché du travail segmenté, dans l'étude des revenus individuels.

D'après l'analyse de la variance, $F_{\text {empirique }}^{*}=13,86$.

Pour un degré de liberté au numérateur de 9 et au dénominateur de 1091, le $\mathrm{F}_{\text {théorique au seuil }}$ de $1 \%$ et de $5 \%$ vaut :

$F_{9, \infty}^{0,01}=2,41$ et $F_{9, \infty}^{0,05}=1,88$.

Quel que soit le seuil, on a donc $F_{\text {empirique }}^{*}>F_{\text {théorique }}$ donc l'hypothèse d'une structure homogène du marché du travail doit être rejetée.

En appliquant le test de Chow, on obtient $F_{\text {empirique }}^{*}=1,65$, que nous comparons au $\mathrm{F}$ théorique $F_{\infty, \infty}^{0,01}=1=F_{\infty, \infty}^{0,05}$.

On retrouve $F_{\text {empirique }}^{*}>F_{\text {théorique }}$.

Il semblerait donc que le marché du travail ne réponde pas à une logique concurrentielle à Antananarivo. Supposer l'existence d'un marché du travail segmenté avec des modes de fixation des revenus différents d'un secteur à l'autre permet alors de mieux décrire le fonctionnement du marché du travail. De fait, les taux de rendement de l'éducation et le poids du type d'éducation dans la détermination des revenus individuels se sont révélés fortement différents d'un secteur à l'autre.

La faiblesse du $\mathrm{R}^{2}$ corrigé, notamment constatée dans le secteur informel, laisse toutefois supposer que les variables retenues dans notre analyse n'expliquent qu'une faible part de la 
variance des revenus dans l'informel ${ }^{23}$. Pour mieux appréhender les rendements de l'éducation, il serait donc nécessaire de bénéficier d'une meilleure connaissance à la fois de la demande de travail et des facteurs politiques et conjoncturels qui caractérisent Madagascar.

\section{Conclusion}

Dans ce papier, nous avons cherché à montrer dans quelle mesure le taux de rendement de l'éducation était surestimé si l'on appliquait le modèle standard de Mincer (1974).

Nous enrichissons alors ce dernier de différentes manières. Aux variables explicatives retenues par l'auteur, l'éducation et l'expérience professionnelle, nous ajoutons le type d'éducation (publique ou privée) ainsi que des caractéristiques socio-économiques. Nous corrigeons le biais de participation au marché du travail selon la procédure d'Heckman (1979) et nous instrumentons le niveau d'éducation, supposé endogène aux revenus.

Un premier modèle de gains est estimé pour les hommes à Antananarivo, en 1997. Le marché du travail est assimilé à un marché concurrentiel. Or, le taux de rendement de l'éducation, significatif dans le modèle de Mincer où il atteignait $10 \%$, perd toute significativité quand nous procédons aux enrichissements préalablement décrits. De plus, alors que l'éducation publique comparée à l'éducation privée réduit significativement la probabilité de travailler, elle n'exerce aucun effet significatif sur les revenus.

Afin de tester la robustesse de ces résultats, nous remettons en question l'hypothèse d'un marché du travail concurrentiel.

Lorsque le modèle de gains est estimé distinctement pour les secteurs formel et informel, il apparaît alors que des actifs occupés, ayant un profil socio-économique et éducatif similaire, perçoivent des rémunérations significativement différentes en fonction de leur appartenance à l'un ou l'autre secteur. Le marché du travail est donc bien segmenté à Antananarivo. Néanmoins, le taux de rendement de l'éducation demeure non significatif dans le secteur formel. Nous l'expliquons par la forte proportion d'instruits qui occupent actuellement un emploi formel mais aussi par une tendance à affecter des individus éduqués à des emplois nécessitant un niveau de qualification inférieur au leur. Dans le secteur informel, en revanche, le taux de rendement de l'éducation atteint $7 \%$ et s'avère robuste aux différentes spécifications du modèle.

23 On retrouve des $\mathrm{R}^{2}$ d'environ 10\% dans les modèles de gains estimés par Morgenstern (1978) sur les Etats-Unis, par exemple. 
Si la durée des études influe sur la rémunération des travailleurs informels, la qualité scolaire joue également un rôle. En effet, dans le secteur informel, l'éducation publique est à l'origine d'un accroissement relatif des revenus. Sur le long terme, soit après un certain nombre d'années d'expérience professionnelle, l'éducation publique fait donc état d'une plus grande efficacité que l'éducation privée dans le secteur informel alors que dans le secteur formel, les deux types d'éducation engendrent des effets non significativement différents sur les revenus. Or, les bénéfices de l'éducation ne se restreignent pas à l'individu mais s'étendent à l'ensemble des agents qui composent l'économie. La présence d'effets externes de l'éducation, combinée à l'efficacité relative de l'éducation publique en termes de revenus individuels, rendrait donc nécessaire l'intervention de l'Etat dans la fourniture de services éducatifs.

Toutefois, le concept de taux de rendement de l'éducation présente un certain nombre de limites qu'il faut garder à l'esprit. D'une part, elle conduit à déterminer des profils de gains à partir de l'observation du revenu de l'individu à un instant t seulement. D'autre part, la théorie du capital humain ne permet pas de comprendre, à elle seule, les écarts de salaires entre des générations différentes. Ceux-ci reflètent des évolutions exogènes du marché du travail et du système scolaire qui sont en partie indépendantes du processus d'accumulation du capital humain. Il en résulte, d'une génération à l'autre, une concurrence plus ou moins intense pour les emplois les mieux rémunérés. Pour cette raison, quand un grand nombre d'individus éduqués entrent sur le marché du travail, le taux de rendement de l'éducation à la date t peut n'être qu'un piètre indicateur de la valeur économique d'une année supplémentaire d'éducation. 


\section{Bibliographie}

Altonji J.G. and Dunn T.A. (1996) : «The Effects of Family Characteristics on the Return to Education », The Review of Economics and Statistics, Vol.LXXVIII, n4, pp.692-704.

Angrist J.D. (1995) : «The Economic Returns to Schooling in the West Bank and Gaza Strip », The American Economic Review, Vol.85, n5, pp.1065-1087.

Angrist J.D. and Lavy V. (1997) : «The Effect of a Change in Language of Instruction on the Returns to Schooling in Morocco », Journal of Labor Economics, Vol.15, n 1 , pp.S48S76.

Angrist J.D. and Krueger A. (1991): «Does Compulsory School Attendance Affect Schooling and Earnings ? », Quaterly Journal of Economics, Vol.106, pp.979-1014.

Arestoff F. (2000) : «Efficacité comparée de l'éduc ation publique et privée. Une application micro-économétrique au cas de Madagascar», Thèse de doctorat, Université Paris XNanterre, Juin.

Arestoff F. et Bommier A. (2000) : ««Efficacité relative des écoles publiques et privées à Madagascar. Etude d'une période de restriction budgétaire», Revue d'Economie du Développement, à paraître.

Ashenfelter O. and Krueger A. (1994) : «Estimating the Returns to Schooling Using a New Sample of Twins », The American Economic Review, Vol. LXXXIV, December, pp.11571173.

Baker M., Benjamin D., Desaulniers A., and Grant M. (1995) : «The Distribution of the Male/Female Earnings Differential, 1970-1990», Canadian Journal of Economics, Vol.XXVIII, n³, pp.479-501.

Becker G. (1975) : «Human Capital», $2^{\text {nd }}$ edition, University of Chicago Press, Chicago.

Bedi A.S. and Garg A. (2000) : «The Effectiveness of Private versus Public Schools : the case of Indonesia », Journal of Development Economics, Vol.61, pp.463-494.

Behrman J.R., Rosenzweig M.R. and Taubman P. (1996) : «College Choice and Wages : Estimates Using Data on Female Twins», The Review of Economics and Statistics, Vol.LXXVIII, n²4, pp.672-685. 
Ben-Porath Y. (1967) : «The Production of Human Capital and the Life Cycle of Earnings », Journal of Political Economy, Vol.75, pp.352-365.

Betts J.R. (1995) : «Does School Quality Matter ? Evidence from the National Longitudinal Survey of Youth», The Review of Economics and Statistics, May, Vol.127, n², pp.231-250.

Boumahdi R. et Plassard J-M. (1992) : «Note à propos du caractère endogène de la variable éducation dans la fonction de gains », Revue Economique, ${ }^{\circ} 1$, Janvier, pp.145-156.

Bowles S. (1972) : « Schooling and Inequality from Generation to Generation», Journal of Political Economy, May/June, pp.219-251.

Brownstone D. and Valletta R.G. (1996) : «Modeling Earnings Measurement Error: A Mutiple Imputation Approach», The Review of Economics and Statistics, Vol.LXXVIII, n4, pp.705-717.

Card D. (1998) : «The Causal Effect of Education on Earnings », in O.Ashenfelter and D. Card (eds), «Handbook of Labor Economics», Vol.3A, Elsevier Science Publishers BV, Netherlands.

Card D. and Krueger A. (1992) : «Does School Quality Matter : Returns to Education and the Characteristics of Public Schools in the United States », Journal of Political Economy, Vol.100, n 1, pp.1-40.

Coquery-Vidrovitch C. (1991) : «L'informel dans les villes africaines : essai d'analyse historique et sociale », in C. Coquery-Vidrovitch et S. Nedele, «Tiers-Monde : l'informel en question», L'harmattan, Paris, pp.171-196.

Coury D. (2000) : «Le marché du travail d'Antananarivo est-il segmenté ? », Mimeo.

Doeringer P.B. (1988) : « Market Structure, Jobs, and Productivity: Observations from Jamaica », World Development, Vol.16, n4, pp.465-482.

Doeringer P.B. and Piore M.J. (1971) : «Internal Labor Markets and Manpower Analysis », Heath Lexington Books, Lexington Massachussets, 214 p.

Gindling T.H. (1991) : «Labor Market Segmentation and the Determination of Wages in the Public, Private-Formal, and Informal Sectors in San José Costa Rica», Economic Development and Cultural Change, Vol.39, n³, pp.584-605. 
Glewwe P. (1996) : «The Relevance of Standard Estimates of Rates of Return to Schooling for Education Policy: A Critical Assessment », Journal of Development Economics, Vol.51, pp.267-290.

Goux D. et Maurin E. (1994) : «Education, expérience et salaire : tendances récentes et évolution de long terme », Economie et Prévision, n¹16, pp.155-178.

Greene W.H. (1997) : «Econometric Analysis », Third Edition, Prentice Hall, New Jersey.

Griliches Z. (1977) : «Estimating the Returns to Schooling : Some Econometric Problems », Econometrica, Vol.45, n ${ }^{\circ}$, January, pp.1-22.

Griliches Z. (1997) : «Education, Human Capital, and Growth : A Personal Perspective », Journal of Labor Economics, Vol.15, n²1, Part.2, January, pp.S330-S344.

Grogger J. (1996) : « School Expenditures and Post-Schooling Earnings : Evidence from High School and Beyond», The Review of Economics and Statistics, Vol.LXXVIII, n4, pp.628-637.

Gronau R. (1973) : «The Effect of Children on the Housewife's Value of Time », Journal of Political Economy, Vol.81, pp. S168-S199.

Harmon C. and Walker I. (1999) : «The Marginal and Average Returns to Schooling in the UK », European Economic Review, Vol.43, pp.879-887.

Heckman J.J. (1974) : «Shadow Prices, Market Wages, and Labor Supply », Econometrica, Vol.42, pp.679-694.

Heckman J.J. (1979) : «Sample Selection Bias as Specification Error», Econometrica, Vol.47, n 1 , pp.153-161.

Heckman J.J. and Polachek S. (1974) : «Empirical Evidence on the Functional Form of the Earnings-Schooling Relationship », Journal of the American Statistical Association, n69, June, pp.350-354.

Heckman J.J. and Hotz V.J. (1986) : «An Investigation of the Labor Market Earnings of Panamanian males: Evaluating Sources of Inequality», Journal of Human Resources, Vol.21, pp.507-542. 
Heckman J.J., Layne-Farrar A. and Todd P. (1996) : «Human Capital Pricing Equations with an Application to Estimating the Effect of Schooling Quality on Earnings », The Review of Economics and Statistics, Vol.LXXVIII, n 4 , pp.562-610.

Ichino A. et Winter-Ebmer R. (1999): «Lower and Upper Bounds of Returns to Schooling : An Exercise in IV Estimation with Different Instruments », European Economic Review, Vol.43, pp.889-901.

Jarousse J.P. et Mingat A. (1986) : «Un réexamen du modèle de gains de Mincer », Revue Economique, Vol.37, $\mathrm{n}^{\circ} 6$.

Knight J.B. and Sabot R.H. (1987) : «The Rate of Return on Educational Expansion», Economics of Education Review, Vol.6, n³, pp.255-262.

Maddala G.S. (1992) : «Introduction to Econometrics », Second Edition, Prentice Hall, New Jersey.

Mincer J. (1974) : «Schooling, Experience and Earnings », New York, National Bureau of Economic Research.

Mincer J. (1993) : «Human Capital and Earnings », in Mincer J., «Studies in Human

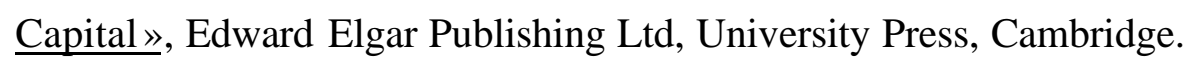

Morgenstern R.D. (1978) : « Direct and Indirect Effects on Earnings of Schooling and Socio-Economic Background », The Review of Economics and Statistics, Vol.LV, $\mathrm{n}^{\circ}$ 2, May, pp. $225-232$.

Murnane R.J., Maynard R.A. and Ohls J.C. (1981) : « Home Resources and Children's Achievement », The Review of Economics and Statistics, Vol.63, n 4 , pp.369-377.

Piore M.J. (1978) : « Dualism in the Labor Market - A Response to Uncertainty and Flux. The Case of France », Revue Economique, Vol.29, n¹ (Janvier).

Projet MADIO (1995) : «L'emploi et les conditions d'activité de la population malgache en 1993 », Etude MADIO, Mars, n95001/E.

Projet MADIO (1997): «L'emploi, le chômage et les conditions d'activité dans l'agglomération d'Antananarivo. Premiers résultats de l'enquête emploi 1997 », Etude MADIO n9728/E, Juin. 
Psacharopoulos G. (1994) : « Returns to Investment in Education: A Global Update », World Development, Vol.22, $\mathrm{n}^{\circ}$, pp.1325-1343.

Ramilison E. (1997) : «Sortie du chômage et durée d'attente d'un emploi formel : étude à partir d'un panel de chômeurs dans l'agglomération d'Antananarivo », Etude MADIO, Avril, $\mathrm{n}^{\circ} 9713 / \mathrm{E}$.

Roubaud F. (1994): «L'économie informelle au Mexique. De la sphère domestique à la dynamique macro-économique », Karthala - ORSTOM, Paris.

Sahn D.E. and Alderman H. (1988) : «The Effects of Human Capital on Wages, and the Determinants of Labor Supply in a Developing Country», Journal of Development Economics, Vol.29, pp.157-183.

Smith P.A. and Metzger M.R. (1998) : «The Return to Education : Street Vendors in Mexico », World Development, Vol.26, n², pp.289-296.

Taubman P. and Wachter M.L. (1986) : «Segmented Labor Markets », in O.Ashenfelter and R.Layard (eds), «Handbook of Labor Economics», Vol.2, Elsevier Science Publishers BV, Netherlands.

Trejo S.J. (1997) : «Why Do Mexican Americans Earn Low Wages? », Journal of Political Economy, Vol.105, n6, pp.1235- 1268. 


\section{Annexes:}

Annexe 1: Estimations de la participation au marché du travail et de l'instrumentation de (Niveau d'études) ${ }^{2}$, respectivement associées aux modèles (c) et (f)

\begin{tabular}{|c|c|c|}
\hline & $\begin{array}{c}\text { Participation au marché du } \\
\text { travail : Probit associé au } \\
\text { modèle (c) }\end{array}$ & $\begin{array}{l}\text { Instrumentation de (Niveau } \\
\text { d'études) })^{2} \text { : MCO associé au } \\
\text { modèle (f) }\end{array}$ \\
\hline Constante & $-1,02^{* *}(0,52)$ & $-130,72^{* * *}(26,52)$ \\
\hline Niveau d'études & $-0,08(0,06)$ & \\
\hline (Niveau d'études) $^{2}$ & $0,004(0,003)$ & \\
\hline Age & $0,12^{* * *}(0,02)$ & $4,75^{* * *}(1,16)$ \\
\hline$(\text { Age })^{2}$ & $-0,002^{* * *}(0,0003)$ & $-0,04^{* * *}(0,01)$ \\
\hline \multicolumn{3}{|l|}{ Type d'éducation } \\
\hline Publique & & $-90,67^{* *}(41,97)$ \\
\hline Privée & & Ref \\
\hline Age*Type d'éducation & & $5,49^{* *}(2,17)$ \\
\hline$(\text { Age })^{2 * t y p e ~ d ' e ́ d u c a t i o n ~}$ & & $-0,08^{* * *}(0,03)$ \\
\hline Revenu des parents & & $0,11^{* * *}(0,03)$ \\
\hline \multicolumn{3}{|l|}{ Religion } \\
\hline Catholique & $0,34^{* * *}(0,13)$ & $3,02(5,5)$ \\
\hline Autre religion & Ref & Ref \\
\hline Nombre de redoublements & $-0,12^{* *}(0,05)$ & \\
\hline Niveau d'études du père & $-0,03(0,02)$ & $7,15^{* * *}(1,11)$ \\
\hline Niveau d'études de la mère & $-0,06^{* *}(0,03)$ & $9,79^{* * *}(1,31)$ \\
\hline \multicolumn{3}{|l|}{ Activité professionnelle du père } \\
\hline Micro-entrepreneur ou à son propre compte & $0,03(0,13)$ & \\
\hline Autre & Ref & \\
\hline \multicolumn{3}{|l|}{ Activité professionnelle de la mère } \\
\hline Micro-entrepreneur ou à son propre compte & $-0,04(0,12)$ & \\
\hline Autre & Ref & \\
\hline \multicolumn{3}{|l|}{ Statut marital } \\
\hline Marié ou en union libre & $0,74^{* * *}(0,16)$ & \\
\hline Autres situations & Ref & \\
\hline \multicolumn{2}{|l|}{ Présence d'enfants de moins de 6 ans } & \\
\hline Oui & Ref & \\
\hline \multicolumn{3}{|l|}{ Non } \\
\hline \multicolumn{3}{|l|}{ Type du premier emploi } \\
\hline Formel & $0,56^{* * *}(0,12)$ & \\
\hline Informel & Ref & \\
\hline Log de la vraisemblance & 278,09 & \\
\hline $\mathrm{R}^{2}$ corrigé (en $\%$ ) & & 35,42 \\
\hline Nombre d'observations & 813 & 636 \\
\hline
\end{tabular}

Source : Enquête SET97, nos propres calculs.

Note $:$ Ref $=$ variable indicatrice de référence.

Seuil de significativité : ***1\%,**5\%,*10\%. Les écarts-types sont notés entre parenthèses. 
Annexe 2: Estimation de la participation au marché du travail et de l'affectation dans le secteur formel, en 1997

\begin{tabular}{|c|c|c|}
\hline & $\begin{array}{c}\text { Participation } \\
\text { au marché du travail } \\
\text { (Probit) } \\
\end{array}$ & $\begin{array}{c}\begin{array}{c}\text { Affectation dans le } \\
\text { secteur formel } \\
\text { (Probit) }\end{array} \\
\end{array}$ \\
\hline Constante & $-1,17^{* * *}(0,38)$ & $-1,98^{* * *}(0,56)$ \\
\hline Age & $0,11^{* * *}(0,02)$ & $0,04^{*}(0,02)$ \\
\hline$(\text { Age })^{2}$ & $-0,001^{* * *}(0,0002)$ & $-0,0004(0,0003)$ \\
\hline \multicolumn{3}{|l|}{ Type d'éducation } \\
\hline Publique & $-0,9^{*}(0,48)$ & $-0,6(0,67)$ \\
\hline Privée & Ref & Ref \\
\hline Age*Type d'éducation & $0,05^{* *}(0,03)$ & $0,02(0,03)$ \\
\hline (Age) $2 *$ Type d'éducation & $-0,0005^{*}(0,0003)$ & $-0,0002(0,0004)$ \\
\hline \multirow{2}{*}{\multicolumn{3}{|c|}{ Religion }} \\
\hline & & \\
\hline Catholique & $0,26^{* * *}(0,07)$ & $-0,14(0,09)$ \\
\hline Autre religion & Ref & Ref \\
\hline Nombre de redoublements & $-0,09^{* * * *}(0,03)$ & \\
\hline Niveau d'études du père & $-0,01(0,01)$ & $0,06^{* * *}(0,02)$ \\
\hline Niveau d'études de la mère & $-0,04^{* *}(0,02)$ & $0,1^{* * *}(0,02)$ \\
\hline \multicolumn{3}{|l|}{ Activité professionnelle du père } \\
\hline Micro-entrepreneur ou à son propre compte & $-0,09(0,08)$ & $-0,07(0,09)$ \\
\hline Autre & Ref & Ref \\
\hline \multicolumn{3}{|l|}{ Activité professionnelle de la mère } \\
\hline Micro-entrepreneur ou à son propre compte & $0,1(0,07)$ & $-0,04(0,08)$ \\
\hline Autre & Ref & Ref \\
\hline \multicolumn{3}{|l|}{ Statut marital } \\
\hline Marié ou en union libre & $0,03(0,08)$ & \\
\hline Autres situations & Ref & \\
\hline \multicolumn{3}{|l|}{$\begin{array}{l}\text { Présence d'enfants de moins de } 6 \text { ans } \\
\text { dans le ménage }\end{array}$} \\
\hline Oui & $0,18^{* *}(0,09)$ & \\
\hline Non & Ref & \\
\hline \multicolumn{3}{|l|}{ Type du premier emploi } \\
\hline Formel & $0,6^{* * * *}(0,07)$ & $0,79^{* * *}(0,14)$ \\
\hline Informel & Ref & Ref \\
\hline \multicolumn{3}{|l|}{ Sexe } \\
\hline Homme & Ref & Ref \\
\hline Femme & $-0,6^{* * *}(0,07)$ & $-0,29^{* *}(0,13)$ \\
\hline \multicolumn{2}{|l|}{ participation au marché du travail $\left(\lambda_{1}\right)$} & $0,06(0,4)$ \\
\hline Log de la vraisemblance & 447,7 & 264,5 \\
\hline Nombre d'observations & 1701 & 1122 \\
\hline
\end{tabular}

Source : Enquête SET97, nos propres calculs.

Note $:$ Ref $=$ variable indicatrice de référence.

Seuil de significativité : ***1\%,**5\%,*10\%. Les écarts-types sont notés entre parenthèses. 
Annexe 3 : Estimation de l'équation standard de Mincer dans les secteurs formel et informel, en 1997

\begin{tabular}{lcc}
\hline & $\begin{array}{c}\text { InY dans le secteur formel } \\
(\mathbf{M C O})\end{array}$ & $\begin{array}{c}\text { InY dans le secteur informel } \\
(\mathbf{M C O})\end{array}$ \\
\hline Constante & $-1,46^{* * *}(0,21)$ & $-1,34^{* * *}(0,19)$ \\
Niveau d'études & $0,09^{* * *}(0,007)$ & $0,08^{* * *}(0,009)$ \\
Age & $0,02^{*}(0,01)$ & $0,01(0,009)$ \\
$(\text { Age })^{2}$ & $-0,00003(0,0001)$ & $-0,00007(0,0001)$ \\
$\mathrm{R}^{2}$ corrigé (en $\left.\%\right)$ & 29,8 & 10,6 \\
Nombre d'observations & 534 & 598 \\
\hline
\end{tabular}

Source : Enquête SET97, nos propres calculs.

Seuil de significativité : $* * * 1 \%, * * 5 \%, * 10 \%$. Les écarts-types sont notés entre parenthèses. 